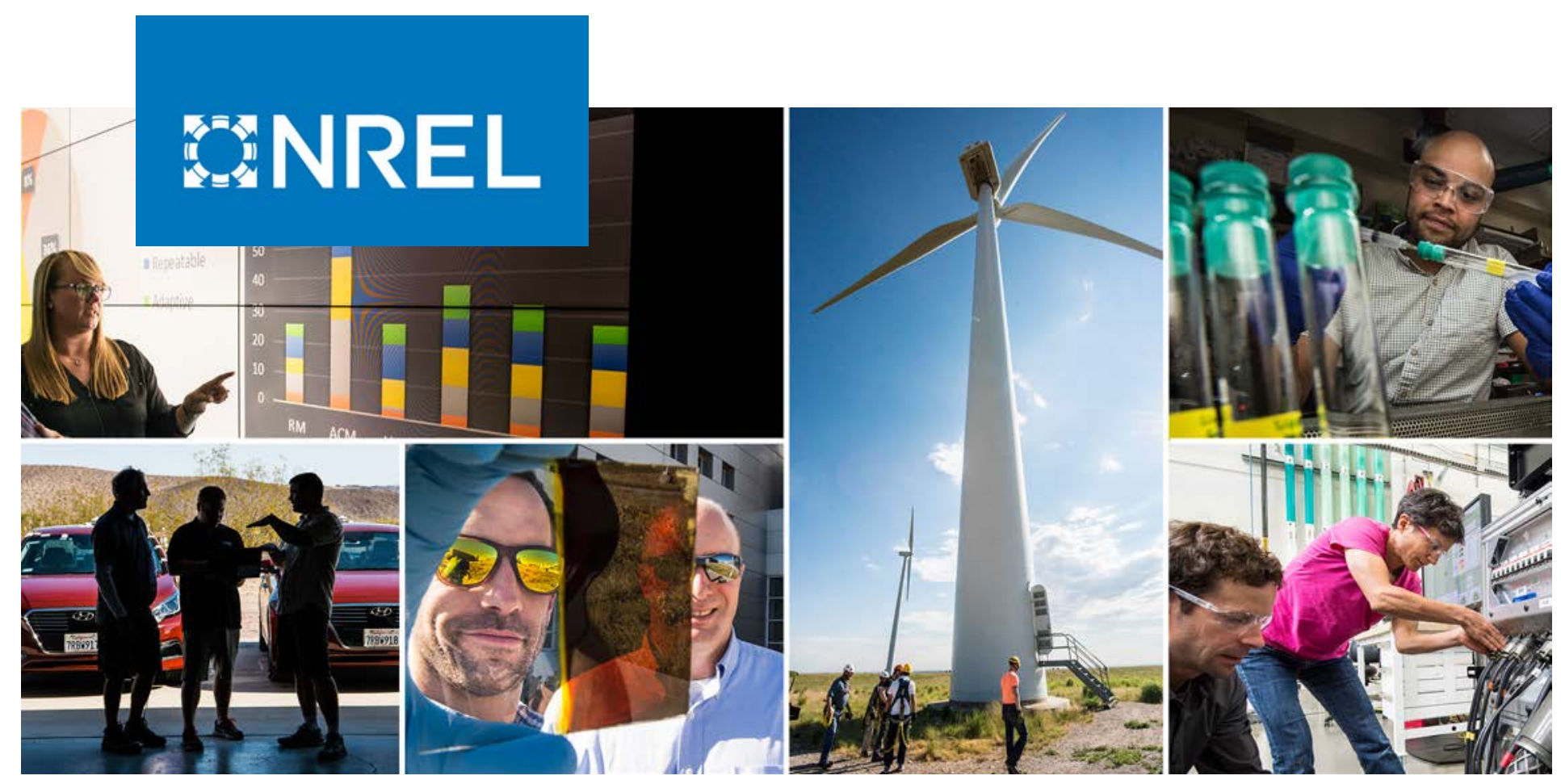

\title{
Assessment of Airfoil Property Sensitivity on Wind Turbine Extreme and Fatigue Loads
}

\section{Preprint}

Kelsey Shaler, Amy N. Robertson, Latha Sethuraman, and Jason Jonkman

National Renewable Energy Laboratory

Presented at the American Institute of Aeronautics and Astronautics

SciTech Forum

San Diego, CA

January 7-11, 2019

NREL is a national laboratory of the U.S. Department of Energy

Office of Energy Efficiency \& Renewable Energy

Operated by the Alliance for Sustainable Energy, LLC

This report is available at no cost from the National Renewable Energy Laboratory (NREL) at www.nrel.gov/publications.
Conference Paper

NREL/CP-5000-72892

January 2019 


\title{
BNREL
}

\section{Assessment of Airfoil Property Sensitivity on Wind Turbine Extreme and Fatigue Loads}

\section{Preprint}

\author{
Kelsey Shaler, Amy N. Robertson, Latha Sethuraman,
} and Jason Jonkman

National Renewable Energy Laboratory

\section{Suggested Citation}

Shaler, Kelsey, Amy N. Robertson, Latha Sethuraman, and Jason Jonkman. 2019.

Assessment of Airfoil Property Sensitivity on Wind Turbine Extreme and Fatigue Loads:

Preprint. Golden, CO: National Renewable Energy Laboratory. NREL/CP-5000-72892.

https://www.nrel.gov/docs/fy19osti/72892.pdf.

NREL is a national laboratory of the U.S. Department of Energy Office of Energy Efficiency \& Renewable Energy Operated by the Alliance for Sustainable Energy, LLC

This report is available at no cost from the National Renewable Energy Laboratory (NREL) at www.nrel.gov/publications.

Contract No. DE-AC36-08GO28308
Conference Paper

NREL/CP-5000-72892

January 2019

National Renewable Energy Laboratory 15013 Denver West Parkway Golden, CO 80401

303-275-3000 • www.nrel.gov 


\section{NOTICE}

This work was authored by the National Renewable Energy Laboratory, operated by Alliance for Sustainable Energy, LLC, for the U.S. Department of Energy (DOE) under Contract No. DE-AC36-08G028308. Funding provided by U.S. Department of Energy Office of Energy Efficiency and Renewable Energy Wind Energy Technologies Office. The views expressed herein do not necessarily represent the views of the DOE or the U.S. Government. The U.S. Government retains and the publisher, by accepting the article for publication, acknowledges that the U.S. Government retains a nonexclusive, paid-up, irrevocable, worldwide license to publish or reproduce the published form of this work, or allow others to do so, for U.S. Government purposes.

This report is available at no cost from the National Renewable Energy Laboratory (NREL) at www.nrel.gov/publications.

U.S. Department of Energy (DOE) reports produced after 1991 and a growing number of pre-1991 documents are available free via www.OSTI.gov.

Cover Photos by Dennis Schroeder: (clockwise, left to right) NREL 51934, NREL 45897, NREL 42160, NREL 45891, NREL 48097, NREL 46526.

NREL prints on paper that contains recycled content. 


\title{
Assessment of Airfoil Property Sensitivity on Wind Turbine Extreme and Fatigue Loads
}

\author{
Kelsey Shaler*, Amy N. Robertson ${ }^{\dagger}$, Latha Sethuraman ${ }^{\ddagger}$, and Jason Jonkman ${ }^{\S}$ \\ National Renewable Energy Laboratory, Golden, CO 80401, USA
}

\begin{abstract}
Wind turbines are designed using a set of simulations to determine the structural loads that the turbine could experience. These structural loads can be significantly influenced by physical turbine parameters. Although significant research and effort has been put into measuring and defining such parameters, limited work has been done to quantify the sensitivity of structural loads to uncertainty in the physical turbine parameters. This paper therefore seeks to assess the sensitivity of different turbine aerodynamic parameters on the resulting ultimate and fatigue loads of the turbine during normal operational conditions. Eighteen different turbine parameters are screened using an Elementary Effects approach with radial points. The results show a high sensitivity of the loads to the blade twist and lift coefficient distributions. To a lesser extent, other turbine parameters that drive loads include the maximum lift coefficient location, blade chord length, and drag coefficient distributions.
\end{abstract}

\section{Nomenclature}

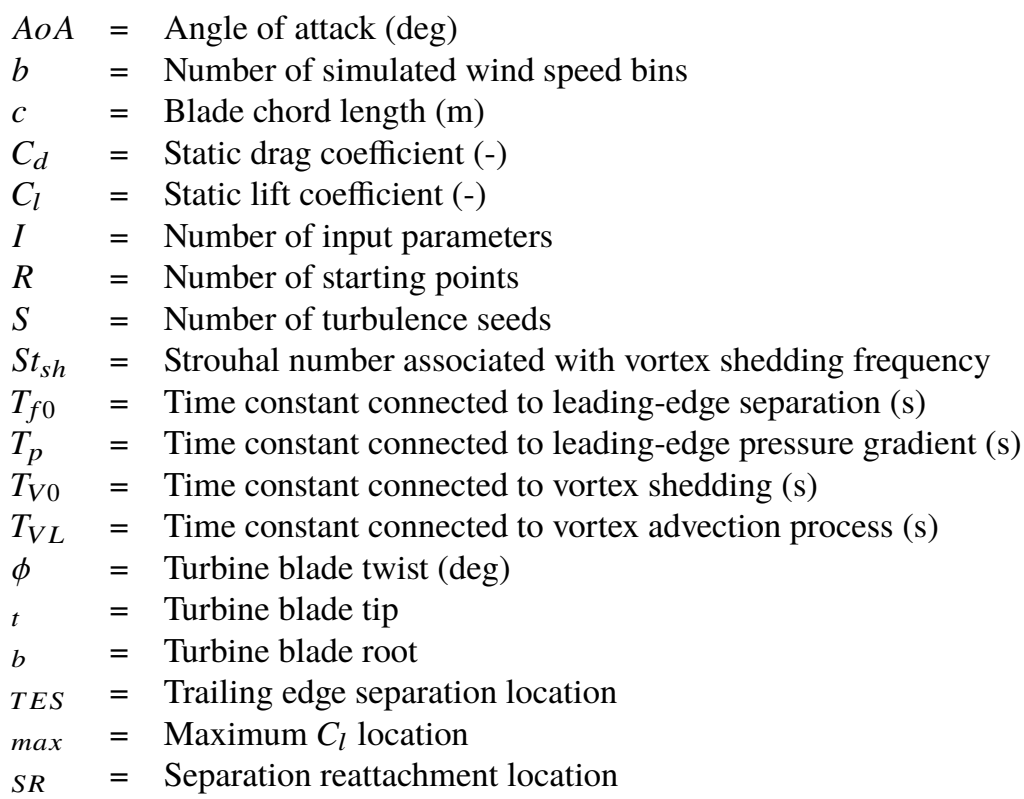

\section{Introduction}

Wind turbine design relies on the ability to accurately predict turbine ultimate and fatigue loads. Loads analysis requires precise knowledge of expected wind inflow conditions as well as turbine structural, aerodynamic, and control properties. However, uncertainty in most parameters is inevitable and it is important to understand the impact such uncertainties have on the resulting fatigue and ultimate loads.

\footnotetext{
*Post-Doctoral Researcher, National Wind Technology Center, kelsey.shaler@ nrel.gov

† Senior Engineer, National Wind Technology Center

†ngineer, National Wind Technology Center

${ }^{\S}$ Senior Engineer, National Wind Technology Center, AIAA Professional Member
} 
Previous work examined the sensitivity of turbine fatigue and ultimate loads to varying wind-inflow parameters [1]. The next step in this work is to characterize load sensitivity to variation in physical parameters of the wind turbine, such as aerodynamic coefficients, structural damping, turbine geometry, masses/inertias, natural frequencies, and so on. This paper focuses specifically on aerodynamic parameters, which are assumed to have the greatest effect on loads relative to other physical parameters. (This assumption is being checked in related follow-on work.) In addition to the blade twist and chord distributions, static lift $\left(C_{l}\right)$ and drag $\left(C_{d}\right)$ coefficient polars and unsteady airfoil aerodynamics are of importance for both load and power prediction. Despite significant work to measure these parameters, considerable uncertainty remains in their prediction. Static lift and drag measurements almost exclusively come from wind tunnel tests of airfoils, which lack three-dimensional and unsteady effects that are instead estimated through the application of semi-empirical engineering models, e.g., rotational augmentation (stall delay) and stall hysteresis [2, 3]. It is widely acknowledged that uncertainty in the aerodynamic parameters can affect the prediction of turbine performance and structural loading [2-4]. Abdallah et al. demonstrated the impact of uncertainty in steady airfoil data on prediction of extreme loads and assessed the correlation between various static coefficent polars [2]. In Damiani, unsteady aerodynamic parameters were tuned for several airfoil sections to match experimental lift and drag unsteady hysteresis loops, but the consequences of parameter variation were not considered [5].

The goal of this work is to assess which aerodynamic parameters, both steady and unsteady, have the greatest influence on fatigue and ultimate loads during normal turbine operation. An Elementary Effects $(E E)$ sensitivity analysis was performed for the aerodynamic input parameters. In this study, the focus is individual parameter sensitivity, though interactions between parameters are considered. The results of this work can be used to 1) rank the sensitivities of different parameters, 2) help establish error bars around the predictions of engineering models during validation efforts, and 3) provide insight to probabilistic design methods.

\section{Sensitivity Analysis Procedure}

An $E E$ method [6-8] was used to assess which aerodynamic parameters have the largest influence on turbine loads. This is a simple methodology for screening parameters and is based on a one-at-a-time approach where each parameter is varied independently while all other parameters remain fixed. The change in response of quantities of interest (QoIs) based on the change in the input parameter is used to compute a derivative, which together with the possible range of the input parameter variation is used to assess the sensitivity of the parameter. This variation and derivative computation is performed several times for each parameter at different points in the hyperspace of all input parameters and is thus considered a global sensitivity analysis method [1]. This method and evaluation process are further discussed by Robertson et al. [1], though some aspects of the method have since been modified.

\section{A. Wind Turbine Model and Tools}

The National Renewable Energy Laboratory (NREL) 5-MW baseline turbine was used in this study as a representative turbine [9] to assess the sensitivity of the input parameters. It is a variable-speed, three-bladed, upwind, horizontal-axis turbine with a hub height of $90 \mathrm{~m}$ and a rotor diameter of $126 \mathrm{~m}$. OpenFAST, an engineering-level modeling approach, was used to simulate a model of the NREL 5-MW wind turbine, allowing for aeroelastic response and turbine operation analysis [10]. AeroDyn, the aerodynamic component of OpenFAST, determines the impact of the turbine wake using induction factors that are computed using blade-element momentum (BEM) theory. Steady and unsteady aerodynamic response were considered. Steady aerodynamic modeling uses static lift and drag curves in the momentum balance to calculate the local induction. Unsteady airfoil aerodynamic modeling accounts for dynamic stall, flow separation, and flow reattachment to calculate the local aerodynamic applied loads. ElastoDyn, a combined multibody and modal structural approach that includes geometric nonlinearities, was used to represent the flexibility of the blades, drivetrain, and tower and compute structural loading, which was used to compute ultimate and fatigue loads. The baseline controller of the NREL 5-MW turbine was enabled using ServoDyn. OpenFAST results were used to assess the change in response QoIs to changes in the physical aerodynamic parameters.

\section{B. Quantities of Interest}

To capture the variability of turbine response that results from parameter variation, several QoIs have been identified. These QoIs are summarized in Table 1 and include blade, drivetrain, and tower loads; blade-tip displacement; and turbine power. Ultimate and fatigue loads were considered for all load QoIs, whereas only ultimate values were considered for blade-tip displacements. The ultimate loads were estimated using the average of the global absolute 
Table 1 Quantities of interest to be examined in sensitivity analysis.

\begin{tabular}{c|ccc}
\hline \hline Quantity of Interest & & Component & \\
\hline Blade-root moments & Out-of-plane bending & In-plane bending & Pitching moment \\
Low-speed shaft moments at main bearing & 0-degree bending & 90-degree bending & Shaft torque \\
Tower-top moment & Fore/aft bending & Side/side bending & Yaw moment \\
Tower-base moment & Fore/aft bending & Side/side bending & \\
Blade-tip displacement & Out-of-plane & \\
& (Ultimate only) & \\
Electrical power & & \\
\hline
\end{tabular}

maximums across all turbulence seeds for a given set of parameter values, whereas the fatigue loads were estimated using damage-equivalent loads [11] of the output response across all seeds for a given set of parameter values. For the bending moments, the ultimate loads were calculated as the largest vector sum of the first two components listed, rather than considering each individually [1].

\section{Sensitivity Analysis}

The $E E$ values were calculated at mean hub-height wind speeds $(B)$ of 8,12 , and $18 \mathrm{~m} / \mathrm{s}$, representing below-, near-, and above-rated wind speeds, respectively. Turbulent wind conditions were generated at each wind speed using TurbSim [12] to generate an International Electrotechnical Commission (IEC) Kaimal turbulence spectra based on wind turbine class $I$ and category $B$ turbulence. Based on a convergence study, 60 turbulence seeds $(S)$ were found to be needed for each parameter space sampling to ensure independence from specific turbulent events (i.e., to ensure that the variation from input parameter changes is distinguishable from the variation from the selection of turbulence seeds). For each simulation, turbine parameters were adjusted as dictated by the $E E$ approach. For this study, 18 aerodynamic input parameters $(I)$ were examined (explained below). This analysis required selecting a number of starting points $(R)$ to be used in the $E E$ approach. For this study, 30 points were found to be sufficient based on a convergence study on the average of the $E E$ value. The total number of 10-minute simulations was $R \times(I+1) \times S \times B=102,600$.

\section{Overview of Elementary Effects}

When considering the $E E$ method, each wind turbine QoI, $Y$, is represented as a function of different characteristics of the model property input parameters, $\mathbf{U}$, as follows:

$$
Y=f\left(U_{1}, U_{2}, U_{3}, \cdots, U_{I}\right)
$$

All input parameters are normalized between 0 (minimum value) and 1 (maximum value). In the general $E E$ approach, for a given sampling of $\mathbf{U}$, the $E E$ value of the $i$ th input parameter is found by varying only that parameter by a normalized amount, $\Delta$ :

$$
E E_{i}=\frac{Y\left(U_{1}, \cdots, U_{i-1}, U_{i}+\Delta, U_{i+1}, \cdots, U_{I}\right)-Y\left(U_{1}, U_{2}, \cdots, U_{I}\right)}{\Delta}
$$

Because of the normalization of $\mathbf{U}$, the elementary effect $\left(E E_{i}\right)$ value can be thought of as the local partial derivative of the output $(Y)$ with respect to the input $\left(U_{i}\right)$, scaled by the range of the input. Thus, the $E E$ value has the same unit as the output, $Y$.

In a radial sensitivity approach, the $E E$ value is calculated for all input parameters at a given point, $r$, in the parameter hyperspace by varying each parameter individually from that point. A representative schematic of this approach is depicted in Figure 1 Each variation is performed for $\pm 10 \%$ of the range over which the parameter may vary $(\Delta= \pm 0.1)$. Note that this is different than the original $E E$ methodology, which creates a trajectory by varying each new parameter from the $\Delta$ point of the previous parameter. This process is repeated for $R$ starting points in the input parameter hyperspace (blue points in Figure 1), creating a set of $R$ different calculations of $E E$ value for each parameter.

\section{E. Elementary Effects Formulas}

This section provides the detailed formulas used to calculate the $E E$ values for both the ultimate and fatigue loads. 


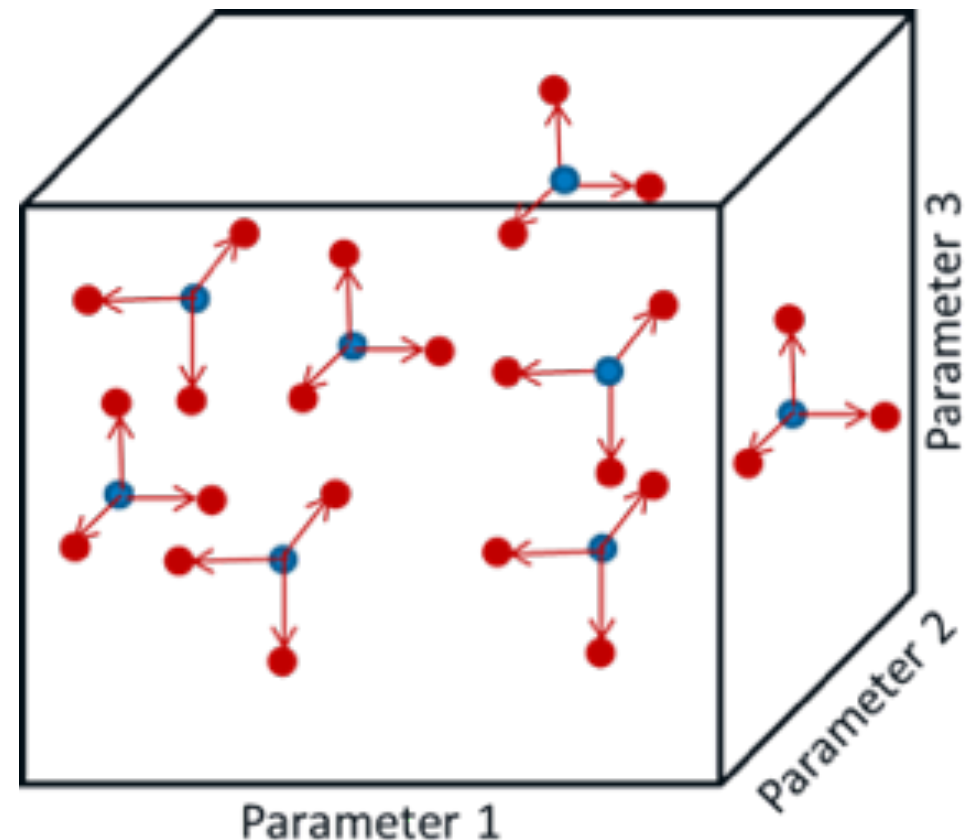

Fig. 1 Representative radial $E E$ approach for three parameters. Blue circles indicate starting points in parameter hyperspace. Red points indicate variation of one parameter at a time.

\section{Ultimate Loads}

When considering the ultimate loads, the standard $E E$ formula was modified by keeping $\mathbf{U}$ and $\Delta$ dimensional (i.e., not nondimensionalizing $\mathbf{U}$ between 0 and 1), multiplying the derivative by the total range of the input for a given wind speed bin, and adding the the IEC-class Ib nominal value of the QoI for the given wind speed bin considered. The latter addition enables the sensitivity of the parameters to be examined consistently across different wind speed bins (because only the single highest ultimate load is of concern, regardless of the wind speed bin). The elementary effect of input parameter $U_{i b}^{r}$ on a certain load response (output), $o$, for starting point $r$ in wind speed bin $b$ is given by:

$$
E E_{o i b}^{r}=\left|\frac{Y_{o}\left(U_{1 b}^{r}, \cdots, U_{i b-1}^{r}, U_{i b}^{r}+\Delta_{i b}, U_{i b+1}^{r}, \cdots, U_{I b}^{r}\right)-Y_{o}\left(U_{1 b}^{r}, U_{2 b}^{r}, \cdots U_{I b}^{r}\right)}{\Delta_{i b}}\left(U_{i b \max }-U_{i b \min }\right)\right|+\bar{Y}_{o b}
$$

where the ultimate load, $Y_{o}()$, is defined as the mean of the absolute maximum of the temporal response load in bin $b$ across $S$ seeds for a certain input parameter $i$ and starting point $r$ :

$$
Y_{o}()=\frac{1}{S} \sum_{s=1}^{S} \operatorname{MAX}\left(\left|Y_{o s}()\right|\right)
$$

and $\operatorname{MAX}\left(\left|Y_{o s}()\right|\right)$ is the absolute maximum of a temporal response load in bin $b$ for starting point $r$ and seed $s$. Additionally, $\Delta_{i b}= \pm \frac{U_{i b \max }-U_{i b \min }}{10}$ and $U_{i b \min }$ and $U_{i b \max }$ are the lower and upper bounds for an input parameter $i$ in bin $b$. The additional term added $\left(\bar{Y}_{o b}\right)$ represents the IEC-class Ib nominal value for the given wind speed bin.

\section{Fatigue Loads}

To compute the fatigue loads, the same basic formulation is used as for the ultimate loads, but the damage equivalent load (DEL) of the temporal response is considered in place of the mean of the absolute maximums:

$E E_{\text {oib }}^{r}=P\left(v_{b}\right)\left|\frac{D E L_{o}^{S T F}\left(U_{1 b}^{r}, \cdots, U_{i b-1}^{r}, U_{i b}^{r}+\Delta_{i b}, U_{i b+1}^{r}, \cdots, U_{I b}^{r}\right)-D E L_{o}^{S T F}\left(U_{1 b}^{r}, U_{2 b}^{r}, \cdots, U_{I b}^{r}\right)}{\Delta_{i b}}\left(U_{i b m a x}-U_{i b m i n}\right)\right|$ 
where $D E L_{o}^{S T F}$ ( ) is the aggregate of the short-term damage equivalent load of output $o$ across all $S$ seeds computed using the NREL postprocessing tool, MLife [11]. DELs are computed without the Goodman correction. The fatigue elementary effect is scaled by $P\left(v_{b}\right)$, which is the Rayleigh probability at the wind speed $v_{b}$ (assuming IEC turbine class I prescription) associated with bin $b$ to compare the fatigue loads consistently across wind speed bins.

\section{Identification of Most Sensitive Inputs}

The $E E$ value is a surrogate for a sensitivity level, and so the higher the $E E$ value calculated for each input parameter across the hyperspace, the more sensitive it is. The approach used here to identify sensitive parameters is to define a threshold over which an individual $E E$ value would be considered to be significant, indicating the sensitivity of the associated parameter. This approach differs from the classical method of determining parameter sensitivity, as discussed in Appendix A The threshold is set individually for each output metric, and is defined as $\overline{E E_{o i b}^{r}}+1.7 \sigma$ where $\overline{E E_{o i b}^{r}}$ is the mean of all $E E$ values across all starting points, all inputs, and all wind speed bins for output $O$ and $\sigma$ is the standard deviation of these $E E$ values. However, because of the separation of $E E$ values between the below-, near-, and above-rated wind speed bins, the ultimate load thresholds are computed using only near- and above-rated results; however, below-rated results are included when the associated $E E$ values occasionally exceed the threshold. Fatigue load $E E$ values are not clearly separated by wind speed; therefore, all wind speeds are used to compute the fatigue load parameter thresholds.

\section{Aerodynamic Parameter Description}

This paper details the process of assessing aerodynamic parameter sensitivities as an initial step towards characterizing overall turbine parameter sensitivity. The aerodynamic properties were varied using 18 input parameters: 3 associated with the blade twist and chord distribution; 10 associated with the static aerodynamic component; and 5 associated with the unsteady aerodynamic properties.

Blade twist and chord distributions were manipulated by specifying a change in the distributions along the blade. Three parameters were defined, associated with changing the chord at the blade tip and root, and the twist at the blade tip. For each of these parameter changes, the associated distribution along the blade was modified linearly such that there was zero change at the opposite end. The root twist was not changed because the blade-pitch angle uncertainties are considered in the full turbine parameter sensitivity study.

Based on the work of Abdallah [2], five static aerodynamic parameters per airfoil were used to perturb the static airfoil lift $\left(C_{l}\right)$ and drag $\left(C_{d}\right)$ polars. However, this study deviates from Abdallah in which parameters were selected, how the parameters were perturbed, and the method of sensitivity analysis. These changes were spurred by the focus on operational loads, as compared to the extreme cases studied by Abdallah. Moreover, the five parameters are defined at the root and tip (10 in total) and varied linearly in between. Additionally, five unsteady aerodynamic properties were considered (uniformly varied across the blade), based on the work of Damiani [5].

\section{A. Steady Airfoil Lift and Drag Curves}

For the steady aerodynamic component, the lift and drag versus angle-of-attack $(A o A)$ curves were modified to examine the sensitivity on resulting loads throughout the wind turbine. The turbine operated in normal operating conditions, and therefore only relevant regions of the curves are modified. To modify the curves, the curves are parameterized using an approach based on one introduced by Abdallah [2]. The approach used here parameterizes the $C_{l}$ and $C_{d}$ curves using five points; these points were perturbed and a spline was fit to the points. The points of interest are:

- beginning of linear $C_{l}$ region - determines the lower limit of the $A o A$ range of interests and were kept constant $\left(\mathbf{C}_{1, \text { lin }}\right)$;

- trailing edge separation (TES) point - AoA location at which $C_{l}$ curve is no longer linear $\left(\mathbf{C}_{\mathbf{l}, \mathbf{T E S}}, \alpha_{\mathbf{T E S}}\right)$;

- AoA location at which $C_{l}$ reaches a maximum and $C_{d}$ begins to increase $\left(\mathbf{C}_{\mathbf{l}, \max }, \mathbf{C}_{\mathbf{d}, \mathbf{0}}, \alpha_{\max }\right)$;

- separation reattachment (SR) point - AoA location at which slope of $C_{l}$ curve is no longer negative $\left(\mathbf{C}_{\mathbf{l}, \mathbf{S R}}, \alpha_{\mathbf{S R}}\right)$;

- intersection of $C_{l}$ and $C_{d}$ - determines the upper limit of the $A o A$ range of interest and was kept constant $\left(\mathbf{C}_{\mathrm{l} \text {,int }}\right)$. The selected points of interest are similar to those selected by Abdallah [2]. A notable difference is the consideration of $C_{d, 0}$ as opposed to $C_{d, 90}$, which is the $C_{d}$ value at $\alpha=90^{\circ} . C_{d, 0}$ was chosen for this study because of the focus on normal operational region, as opposed to the extreme conditions considered by Abdallah [2]. The three variable points of interest were perturbed by a percentage of the default value. The perturbations and correlations are depicted in Figure 2] From Abdallah [2], the TES, max, and SR $C_{l}$ values for an individual airfoil have a correlation to one another 


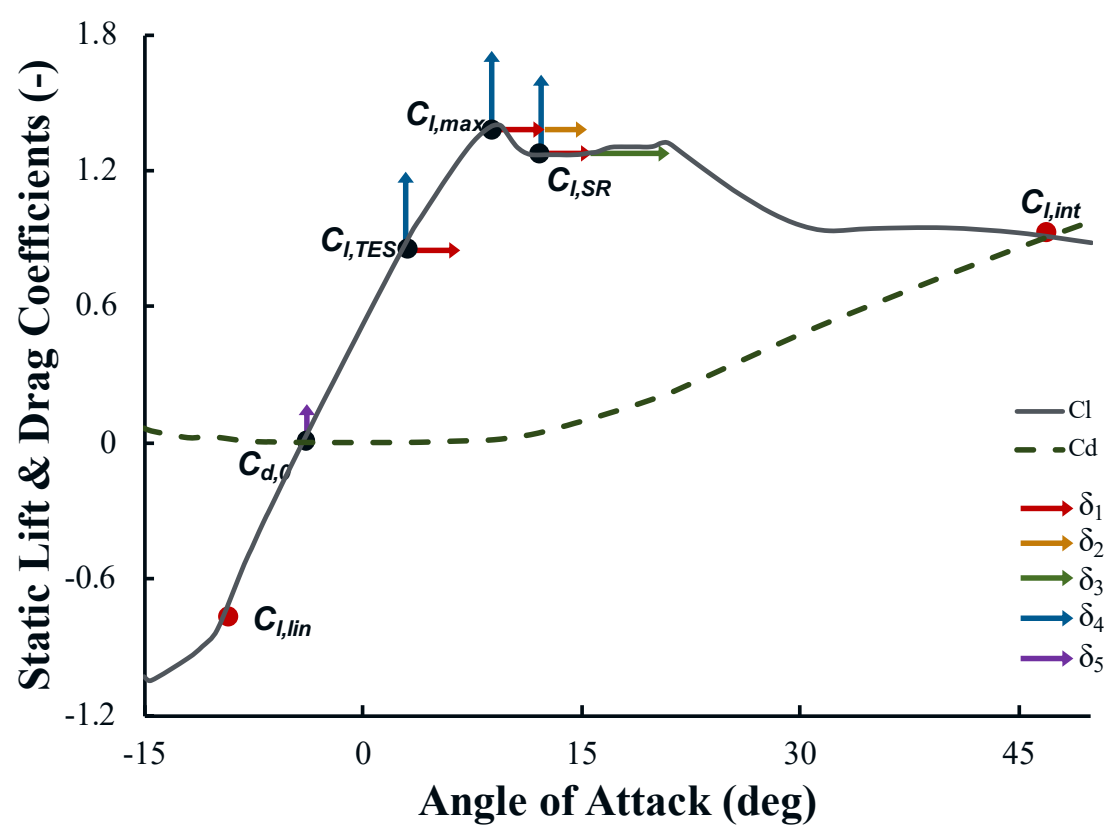

Fig. 2 Perturbation of points of interest in $C_{l}$ and $C_{d}$ curves.

of 0.9. Thus, all $C_{l}$ values are perturbed collectively, using the same percentage $\left(\delta_{4}\right)$. The $A o A$ values are less correlated and so are therefore perturbed independently of one another. However, to ensure that nonphysical relative values are not reached, all $A o A$ values are perturbed by the same base percentage $\left(\delta_{1}\right)$, and then an additional independent variation of a smaller value was added ( $\delta_{2}$ and $\left.\delta_{3}\right)$ for $\alpha_{\mathbf{m a x}}$ and $\alpha_{\mathbf{S R}}$, respectively. The $C_{d, 0}$ was also perturbed $\left(\delta_{5}\right)$.

$C_{l}$ and $C_{d}$ curves are altered for each airfoil. However, instead of specifying $\delta$ values for each airfoil, these values are specified at the root and tip airfoils, excluding the cylindrical airfoils at the base. Perturbation values for the interior airfoils are computed from a linear fit of the end point values. The method of developing the new curves for each airfoil is detailed here:

1) $A o A$ deltas are applied to the original $A o A$ values via Equations 68 .

$$
\begin{gathered}
\alpha_{T E S, \text { new }}=\alpha_{T E S, \text { orig }}+\alpha_{T E S, \text { orig }} \delta_{1} \\
\alpha_{\text {max,new }}=\alpha_{\text {max,orig }}+\alpha_{\text {max,orig }}\left(\delta_{1}+\delta_{2}\right) \\
\alpha_{S R, \text { new }}=\alpha_{S R, \text { orig }}+\alpha_{S R, \text { orig }}\left(\delta_{1}+\delta_{3}\right)
\end{gathered}
$$

2) The new $A o A$ values are fit to the nearest existing $A o A$ value on the curve. The $A o A$-value resolution is fine enough that all perturbations are captured, though not precisely. This approach may need to be adjusted if the perturbations were to decrease.

3) For all new $A o A$ values, the change in $C_{l}$ between the original $C_{l}$ value $\left(C_{l, \text { orig }}\right)$ and the $C_{l}$ curve value at the new $A o A\left(C_{l, o r i g+}\right)$ is computed via Equation 9 .

$$
\epsilon=C_{l, \text { orig+ }}-C_{l, \text { orig }}
$$

4) The total change in $C_{l}$ is then computed via:

$$
C_{l, \text { diff }}=\delta_{4} C_{l, \text { orig }}-\epsilon
$$

This ensures that if $\delta_{4}=0$, the final $C_{l, \text { new }}$ value is equivalent to that of the original curve.

5) For $C_{l}$ perturbation, the end points are located at the $A o A$ s associated with the beginning of the linear $C_{l}$ region $\left(C_{l \text { lin }}\right)$ and $A o A=90^{\circ}$; as these are fixed points, they have $C_{l, \text { diff }}=0$. The $C_{l}$ curve is replaced by a line between 

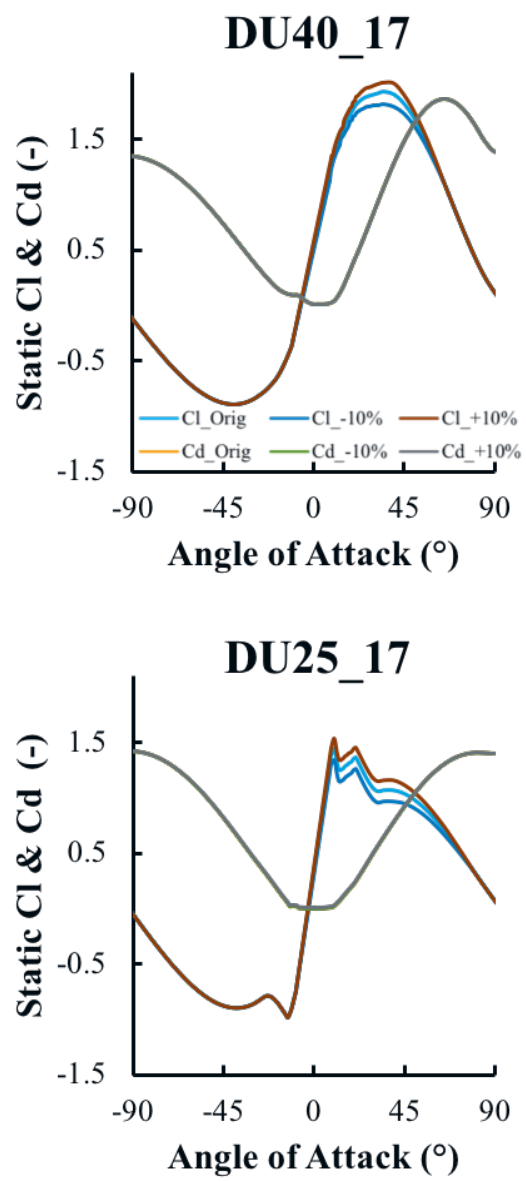

DU35_17

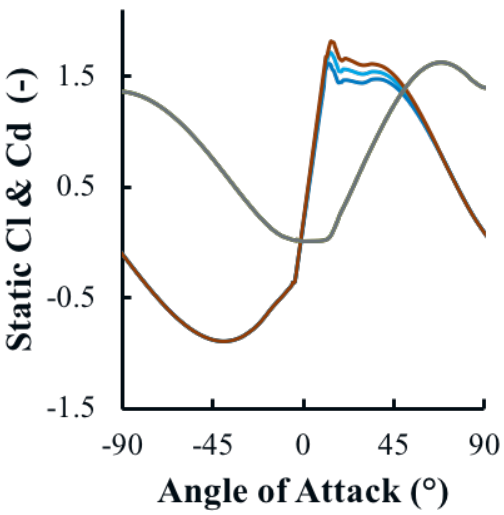

DU21_17

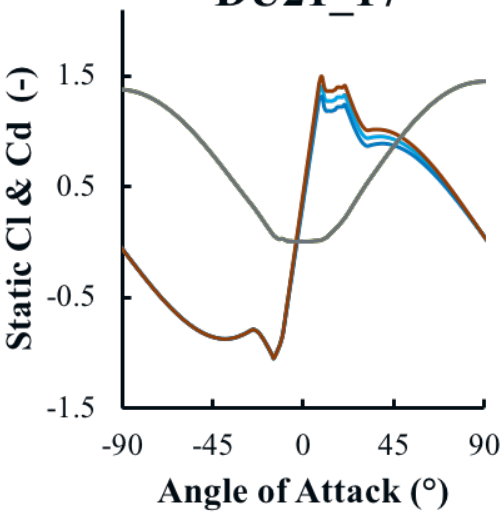

DU30_17

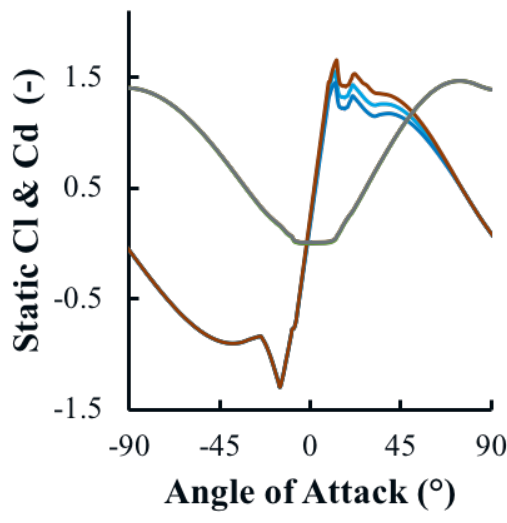

NACA64_A17

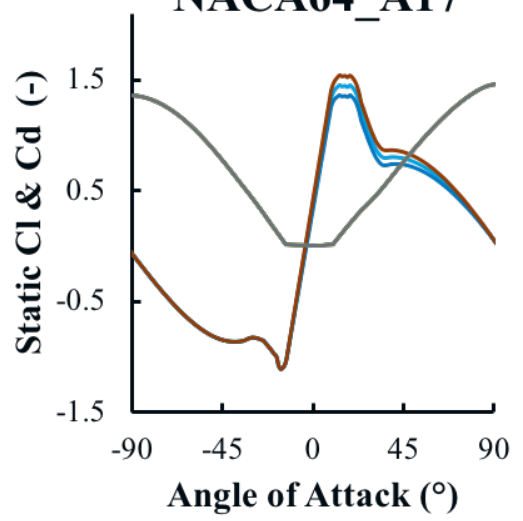

Fig. 3 Sample original and perturbed $C_{l}$ and $C_{d}$ curves for each airfoil section used in the NREL 5MW reference turbine. Perturbed values represent $\pm 10 \%$ of the specified range for each parameter.

$\left(A o A_{l i n}, C_{l \text { lin }}\right)$ and $\left(A o A_{T E S}, \delta_{4} C_{l_{T E S}}\right)$. A piece-wise linear spline - representing perturbations about the original curve - is constructed between the points $\left(A o A_{T E S_{\text {new }}}, \delta_{4} C_{l_{T E S}}\right) ;\left(A o A_{\text {max }_{\text {new }}}, \delta_{4} C_{l_{\text {max }}}\right) ;\left(A o A_{S R_{\text {new }}}, \delta_{4} C_{l_{S R}}\right)$; and $\left(90^{\circ}, 0\right)$.

6) The $C_{l, \text { diff }}$ values calculated from the spline fit are added to the original $C_{l}$ curve.

$$
C_{l, \text { new }}=C_{l, \text { orig }+}+C_{l, \text { diff }}
$$

Several modified $C_{l}$ and $C_{d}$ curves are shown for each airfoil section in Figure 3 . Note that $C_{d}$ curves are perturbed, but by a very small amount not visible in the plots. These perturbations result in modified $C_{l}$ curves that maintain the primary characteristics of the original curve, but differ in both magnitude and feature location. A similar process was followed for modifying the $C_{d}$ curves, wherein the $C_{d}$ value corresponding to $\alpha=0^{\circ}\left(C_{d 0}\right)$ is perturbed by a specified value $\left(\delta_{5}\right)$ in the same manner as the $C_{l}$ values. A piece-wise linear spline is then fit between $\left(-90^{\circ}, C_{d,-90)},\left(0^{\circ}, C_{d, 0}\right)\right.$, and $\left(90^{\circ}, C_{d, 90}\right)$ and added to the original $C_{d}$ curve. $C_{d 0}$ is constrained to not go below 0.

\section{B. Unsteady Airfoil Aerodynamics}

There are several unsteady airfoil aerodynamic parameters that can be modified in OpenFAST. By expert opinion [13], several of these parameters have been identified as having the largest potential variability or impact on turbine response and are therefore included in this study. Several of the parameters in the Beddoes-Leishman-type unsteady airfoil aerodynamics model used here are derivable from the (perturbed) static lift and drag polars (so in this study, when the lift and drag polars are perturbed, the associated Beddoes-Leishman unsteady airfoil aerodynamic parameters are 
Table 2 Parameter value ranges of aerodynamic properties.

\begin{tabular}{c|ccccccccccccc}
\hline \hline Param. & $\begin{array}{c}\phi_{\text {tip }} \\
(d e g)\end{array}$ & $\begin{array}{c}\mathbf{c}_{\text {base }} \\
(m)\end{array}$ & $\begin{array}{c}\mathbf{c}_{\text {tip }} \\
(\mathrm{m})\end{array}$ & $\begin{array}{c}\mathbf{T}_{\mathbf{f 0}} \\
(s)\end{array}$ & $\begin{array}{c}\mathbf{T}_{\mathbf{V 0}} \\
(s)\end{array}$ & $\begin{array}{c}\mathbf{T}_{\mathbf{p}} \\
(s)\end{array}$ & $\begin{array}{c}\mathbf{T}_{\mathbf{V L}} \\
(s)\end{array}$ & $\begin{array}{c}\mathbf{S t}_{\mathbf{s h}} \\
(-)\end{array}$ & $\begin{array}{c}\alpha_{\mathbf{T E S}, \mathbf{t r}} \\
\left({ }^{\circ}\right)\end{array}$ & $\begin{array}{c}\alpha_{\text {max,tr }} \\
\left({ }^{\circ}\right)\end{array}$ & $\begin{array}{c}\alpha_{\mathbf{S R}, \mathbf{t r}} \\
\left({ }^{\circ}\right)\end{array}$ & $\begin{array}{c}\mathbf{C}_{\mathbf{l}, \mathbf{t r}} \\
(-)\end{array}$ & $\begin{array}{c}\mathbf{C}_{\mathbf{d}, \mathbf{t r}} \\
(-)\end{array}$ \\
\hline Nom. & 0.106 & 3.542 & 1.419 & 6.5 & 8 & 1.35 & 16.5 & 0.245 & $v a r$. & $v a r$. & var. & var. & var. \\
Min. & -1.894 & 3.1878 & 1.2771 & 3 & 1 & 1 & 11 & 0.19 & $-20 \%$ & $-8 \%$ & $-15 \%$ & $-26 \%$ & $-100 \%$ \\
Max. & 2.106 & 3.8962 & 1.5609 & 10 & 15 & 1.7 & 22 & 0.3 & $+20 \%$ & $+8 \%$ & $+15 \%$ & $+26 \%$ & $+100 \%$ \\
\hline Ref. & {$[14]$} & {$[15]$} & {$[15]$} & {$[5]$} & {$[5]$} & {$[5]$} & {$[5]$} & {$[5]$} & {$[2]$} & {$[2]$} & {$[2]$} & {$[2]$} & {$[16]$} \\
\hline \hline
\end{tabular}

perturbed as well). Additionally, there are several other parameters associated with unsteady aerodynamics that are included in OpenFAST. These parameters are:

- $\mathbf{T}_{\mathbf{f} \mathbf{0}}$ - time constant connected to leading-edge separation of the airfoil;

- $\mathbf{T}_{\mathbf{V 0}}$ - time constant connected to vortex shedding;

- $\mathbf{T}_{\mathbf{p}}$ - time constant connected to the leading-edge pressure gradient;

- $\mathbf{T}_{\mathbf{V L}}$ - time constant connected to the vortex advection process;

- $\mathbf{S t}_{\mathbf{s h}}$ - Strouhal number associated with the vortex shedding frequency.

These quantities are varied over the ranges detailed in the following section and are constant across the blade.

\section{Parameter Ranges}

To assess the sensitivity, a range needs to be assigned over which the parameters could feasibly vary. The ranges considered for each parameter are summarized in Table $2 \delta$ ranges are given for steady parameters and value ranges are given for unsteady parameters. All $\delta$ values are based on coefficient of variance (COV) values from Abdallah [2], defined in Equation 12.

$$
\operatorname{COV}=\frac{\sigma}{\bar{x}}
$$

Here, $\sigma$ is the standard deviation of the quantity and $\bar{x}$ is the default (original) value per airfoil. Most ranges are taken to be $\pm 2 \operatorname{COV} \bar{x}$; this is equivalent to including $2 \sigma$ on either side of the mean, thus including the $95 \%$ quantile of the parameter range. Note that actual range values differed per airfoil as each airfoil has different default parameter values. As previously mentioned, $C_{l}$ values around stall were found to have a correlation coefficient of 0.9 and are therefore prescribed the same $\delta$ value. AoA values have lower correlation so were initially assigned independent $\delta$ values. However, because of the significant range of allowable values, this method could potentially result in nonphysical lift curves, e.g., $C_{l, \max }$ occurring at a lower $A o A$ than $C_{l, T E S}$. To alleviate this problem, all $A o A$ values are perturbed by $\alpha_{T E S, t r}$. Then, $\alpha_{\max }$ and $\alpha_{S R}$ are additionally perturbed by half $C O V \bar{x}$.

Unsteady airfoil ranges were found by comparing recommended values in Damiani [5] and default values for the airfoil family used with the NREL 5-MW turbine. Ranges were chosen to be the minimum and maximum of all values from these sources. Because these parameters vary the same for each airfoil, they are presented as numeric ranges instead of a percent change. Blade chord and twist ranges were chosen using the work of Loeven and Bihl, who identified changes in blade chord and twist based on uncertainty in aerodynamic loading, icing, or wear of the blades [15].

\section{Results}

The $E E$ value was calculated for all 18 input parameters at 30 different points in the input parameter hyperspace. The $E E$ values across all input parameters, input hyperspace points, and wind speed bins were examined for all output metrics for the ultimate and fatigue loads. For each output parameter, the number of times an $E E$ value exceeded the threshold for a given output metric was tallied. The resulting tallies are shown in Figure 4 , with the ultimate load tally on the left and the fatigue load tally on the right. Note that nearly twice as many significant events were counted for fatigue loads; less significant events were counted for ultimate loads because of the limited threshold exceedence in the below-rated wind speeds. The percentage that each relevant input parameter contributed to the total significant event count is summarized in Table 3 . Both ultimate and fatigue turbine loads are most sensitive to the $C_{l}$ distribution at the outboard section of the blade $\left(C_{l, t}\right)$, which accounted for nearly half of all significant events. Turbine loads are also sensitive to the $C_{l}$ distribution at the inboard section of the blade $\left(C_{l, b}\right)$ and twist distribution $(\phi)$, both of which contributed nearly a quarter of the ultimate and fatigue load significant event counts. Though these results are expected, 

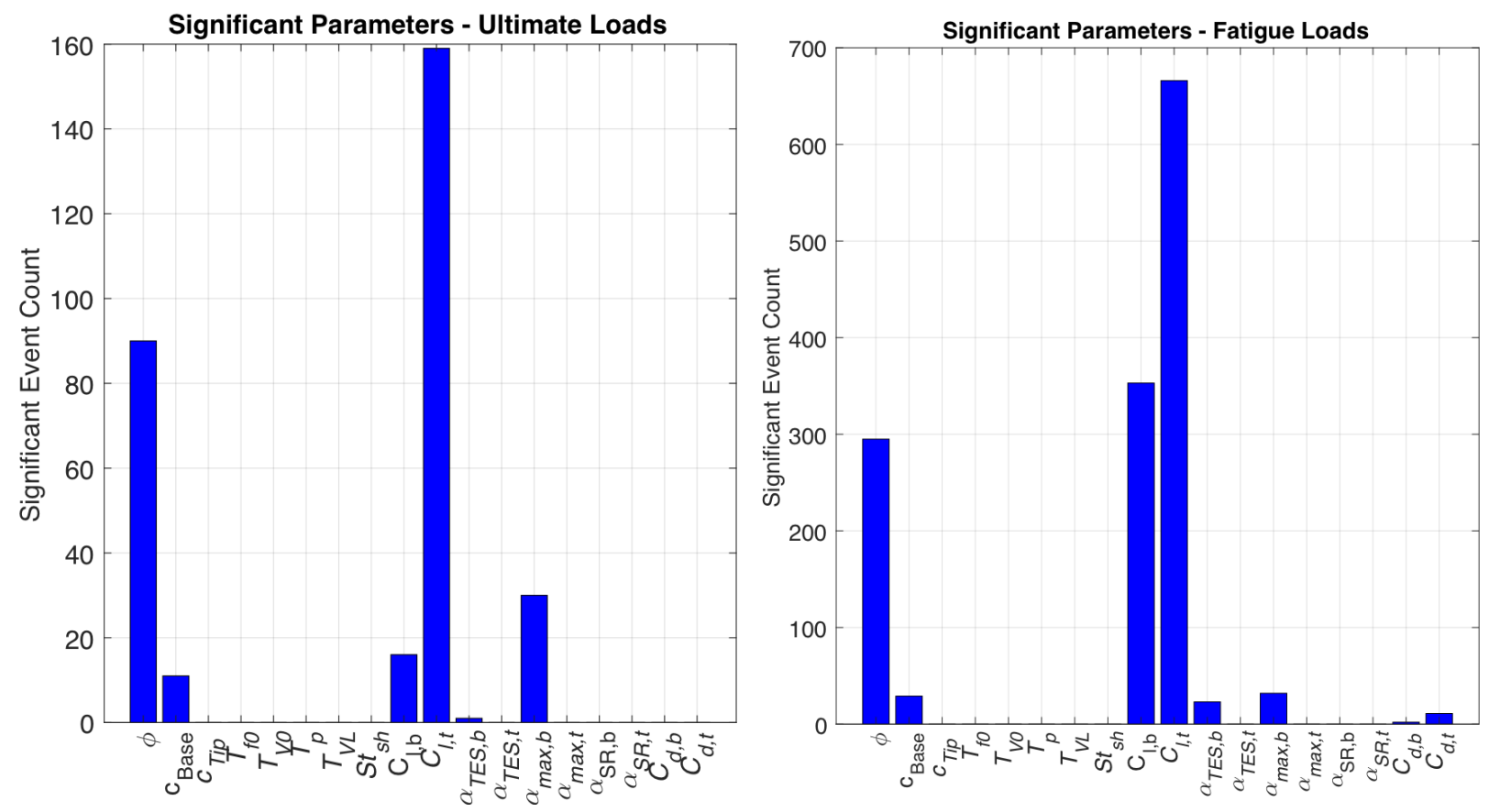

Fig. 4 Identification of significant parameters based on both ultimate (left) and fatigue loads (right). Significant events are defined by the number of outliers identified across all outputs for all wind speed bins, input parameters, and simulation points.

Table 3 Percentage of contribution to total number of significant events for both ultimate and fatigue loads.

\begin{tabular}{l|ccccccccc}
\hline \hline & $C_{l, t}$ & $C_{l, b}$ & $\phi$ & $\alpha_{\max , b}$ & $c_{b}$ & $\alpha_{T E S, b}$ & $C_{d, t}$ & $C_{d, b}$ & $c_{t}$ \\
\hline UIt. Load (\%) & 43.7 & 19.3 & 24.4 & 7.5 & 3.6 & 0.5 & 0.5 & 0.2 & 0.2 \\
Fat. Load (\%) & 47.7 & 24.9 & 22.9 & 2.5 & 0.2 & 0.7 & 0.9 & 0.2 & 0.1 \\
\hline
\end{tabular}


their relevant importance is likely a new finding. Other input parameters that were found to affect turbine load sensitivity are maximum $A o A$ and chord length at the inboard section of the blade ( $\alpha_{\max , b}$ and $c_{b}$, respectively). There is also minor sensitivity to TES $A o A$ at the inboard section of the blade $\left(\alpha_{T E S, b}\right)$; chord length at the outboard section $\left(c_{t}\right)$; and the $C_{d}$ distribution $\left(C_{d, t}\right.$ and $\left.C_{d, b}\right)$. The $A o A$ s at the inboard section of the blade are likely more important than at outboard section due to the higher likelyhood of the inboard section operating with higher AoA near stall. Additionally, the range of $A o A$ values at the inboard section is larger than at the outboard section because the nominal inboard $A o A$ values are higher, which could contribute to greater sensitivity.

Exceedance probability of the $E E$ values for all outputs are plotted in Figures 5 and 6 for the ultimate and fatigue load metrics, respectively. Each plot contains all calculated $E E$ values for a given output colored by wind speed bin. The threshold used to identify significant $E E$ values (as described in Section III.E.3) is shown in each plot as a solid black line. All points above the threshold line indicate a significant event and are included in the outlier tally for each output. Note that although electric power is shown, it is not used in the outlier tally because its variation is strictly limited by the turbine controller rather than by other turbine properties. As shown in Figure 5, ultimate load $E E$ values are grouped by wind speed bin for all outputs with the exception of blade-root pitching moment. These results highlight the unequal distribution of outliers resulting from each turbine output parameter. Most notably, blade-root pitching moment accounts for $21 \%$ of the total ultimate load significant events, whereas rotor torque accounts for only $1 \%$. This suggests that it may be better to tailor the threshold for each output, but this was deemed overly complicated for this first pass at assessing the sensitivity. Additionally, for a given output, it is typical for all ultimate load significant events to occur for either the near- or above-rated wind speeds. However, fatigue load $E E$ values more evenly distributed across wind speed bins, as shown in Figure 6 The lower significant event counts for ultimate loads is a result of the segregated nature of the ultimate load $E E$ values, as opposed to the more evenly distributed nature of the fatigue load $E E$ values. In fact, unlike ultimate load $E E$ values, a large percentage of significant events result from below-rated wind speed cases (because of the higher probability of low wind speed conditions). Additionally, there is a more even distribution of outliers resulting from each turbine output parameter, with the highest percentage (12\%) resulting from the blade-root out-of-plane (OoP) bending moment and the lowest percentage $(3.3 \%)$ resulting from the blade-root pitching moment.

These points are further highlighted by computing $E E$ value histograms for ultimate and fatigue load outputs. Ultimate load histograms are shown in Figure 7. Here, $E E$ values are again colored by wind speed and the black vertical line represents the threshold for each output. The sharp separation of ultimate load $E E$ values between wind speed bins is again evident. A zoomed-in view of the lower count values is shown in Figure 8. The more evenly distributed nature of the fatigue load $E E$ values is further highlighted in the histogram plot depicted in Figure 9 and zoomed in Figure 10. Unlike ultimate load $E E$ values, more than one wind speed bin contributes to the outlier count for each output parameter. Additionally, the more equal output parameter sensitivity is shown in Figure 10 by the distribution of outliers across all output parameters.

Histogram plots of blade-root bending moment $E E$ values are shown in Figures 11-Figures 14 In each figure, wind speed bins are displayed in different plots and $E E$ value histograms showing the contribution from all input parameters are shown in each plot. Ultimate load $E E$ values are shown in Figure 11, with a zoomed-in view of the lower count values shown in Figure 12. These plots highlight the separation of wind speed bin $E E$ values. As shown in Figure 12 . only occurrences of $\phi$ and $C_{l, t}$ lie beyond the threshold limit. Fatigue load $E E$ values are shown in Figure 13 , with a zoomed-in view of the lower count values shown in Figure 14 These plots highlight the more even distribution of threshold-exceeding $E E$ values, as opposed to the ultimate load $E E$ values.

The behavior of blade-root loads are examined in more detail by plotting the exceedance probability distinctly for each input parameter, as shown in Figure 15. Here, all input parameters are plotted independently of each other to compare the behavior between parameters. Each line represents a different input parameter, with each point representing a different location in the hyperspace. Highlighted in these plots is the contribution of the individual input parameters to the outlier counts. For blade-root bending ultimate moment $E E$ values, blade twist and $C_{l, t} E E$ values in the near-rated wind speed bin are beyond the threshold for every point in the hyperspace. No other points from other input parameters cross the threshold. For blade-root OoP bending fatigue moment $E E$ values, the threshold is exceeded by blade twist, $C_{l, t}$, and $C_{l, b} E E$ values in the below- and near-rated wind speed bins. The threshold is exceeded for all $C_{l, t}$ points in the below- and near-rated wind speed bins and all blade twist points in the near-rated wind speed bin. However, for all other relevant input parameters, only certain points in the hyperspace result in threshold exceedance. This indicates that, for certain loads and input parameters, the sensitivity of the turbine is dependent on the combination of turbine parameter values. These results can be used in future studies to more thoroughly investigate the hyperspace to determine how input parameter value combinations contribute to turbine sensitivity. 

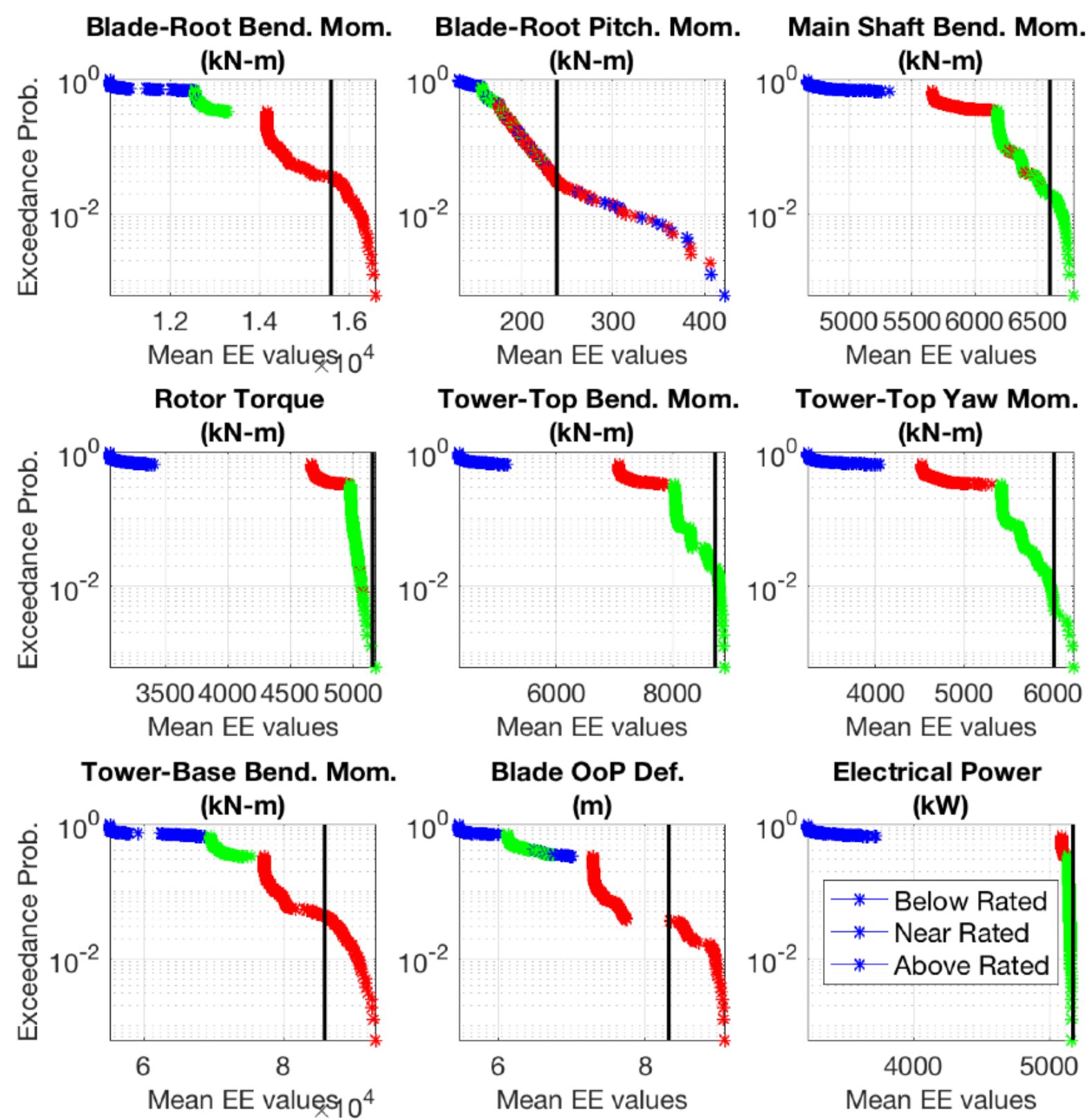

Fig. 5 Exceedance probability of ultimate load $E E$ values across all wind speed bins, input parameters, and simulation points for all outputs. The black line represents the defined threshold by which outliers are counted for each output. Color indicates wind speed bin (blue=below rated, red=near rated, green=above rated). 


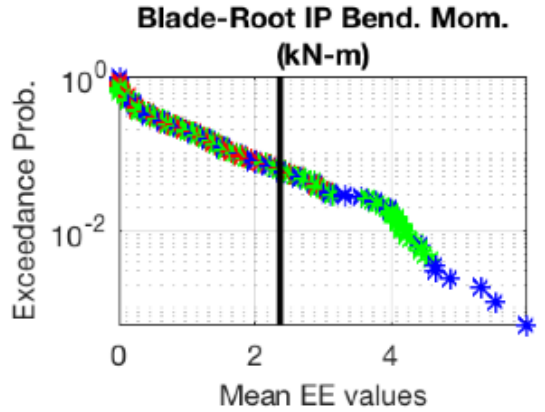

Main Shaft Bend. Mom. 0 deg

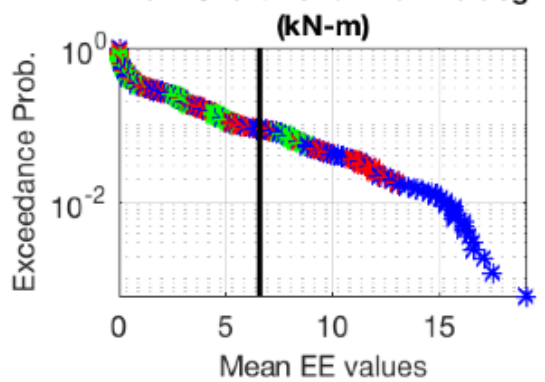

Tower-Top FA Bend. Mom.

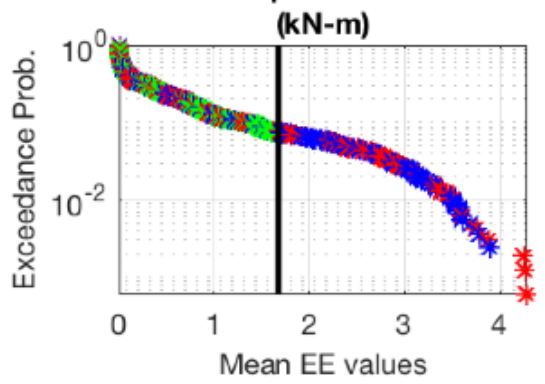

Tower-Base FA Bend. Mom.

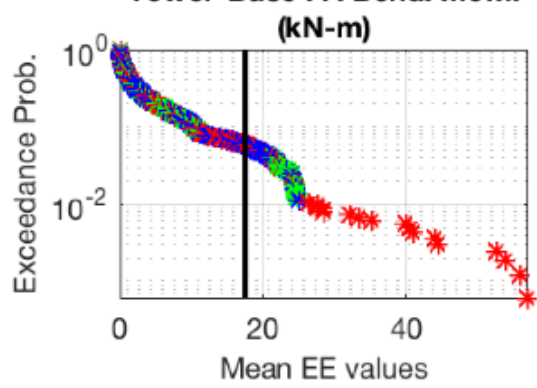

Blade-Root OoP Bend. Mom. (kN-m)

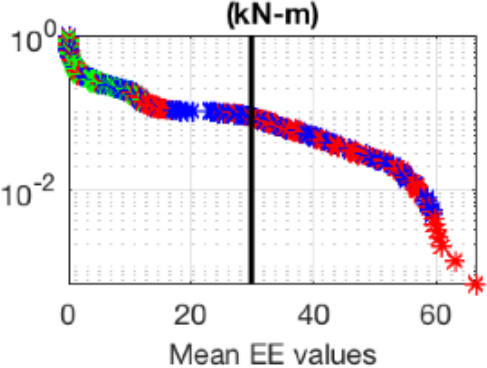

Main Shaft Bend. Mom. 90 deg (kN-m)

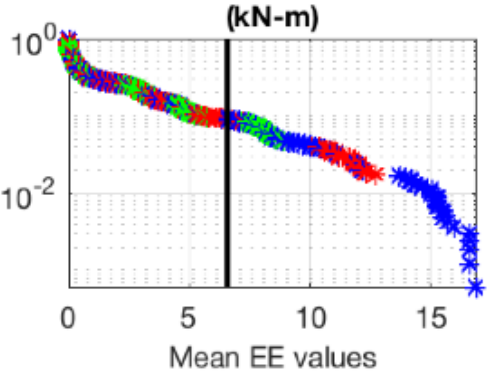

Tower-Top SS Bend. Mom.

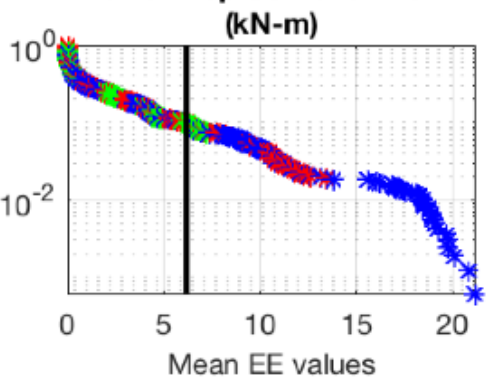

Tower-Base SS Bend. Mom.

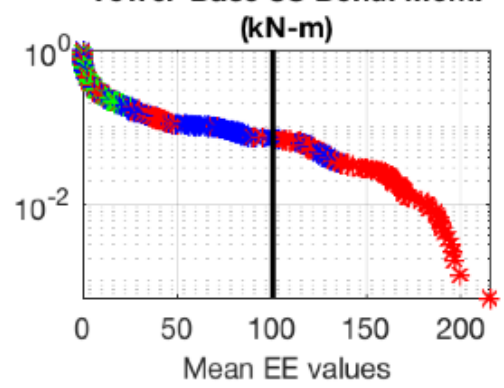

Blade-Root Pitch. Mom.

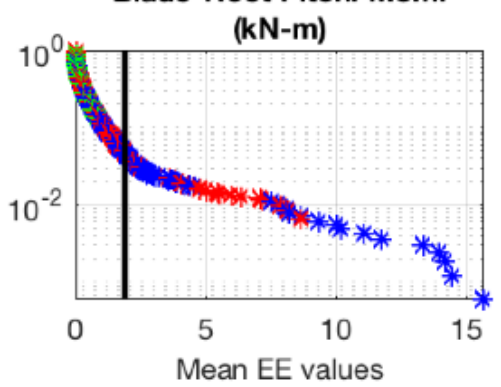

Rotor Torque (kN-m)

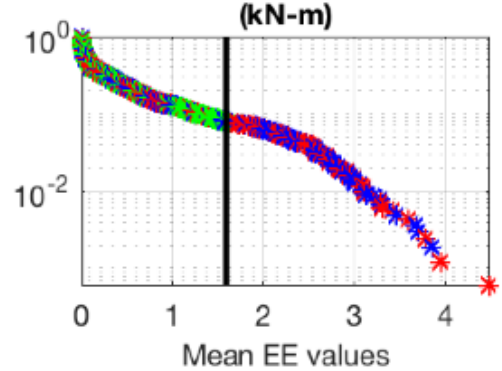

Tower-Top Yaw Mom.

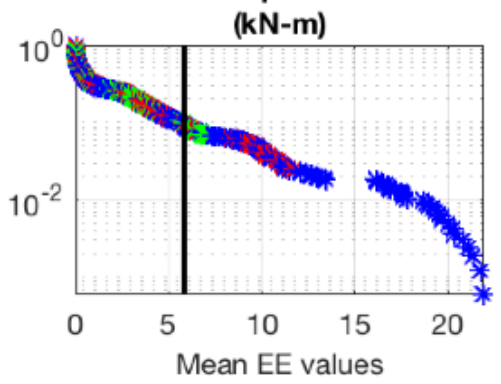

Electrical Power (kW)

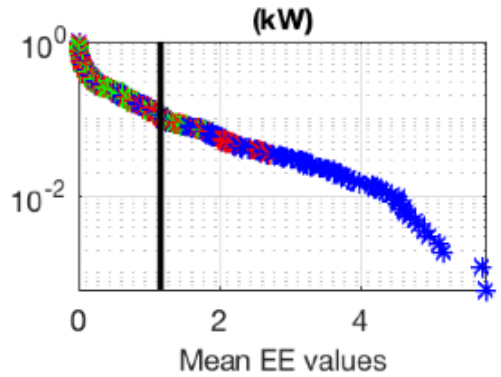

Fig. 6 Exceedance probability of fatigue load $E E$ values across all wind speed bins, input parameters, and simulation points for all outputs. The black line represents the defined threshold by which outliers are counted for each output. Color indicates wind speed bin (blue=below rated, red=near rated, green=above rated). IP=inplane; FA=fore-aft; and SS=side-to-side. 
Blade-Root Bend. Mom. Blade-Root Pitch. Mom. Main Shaft Bend. Mom.
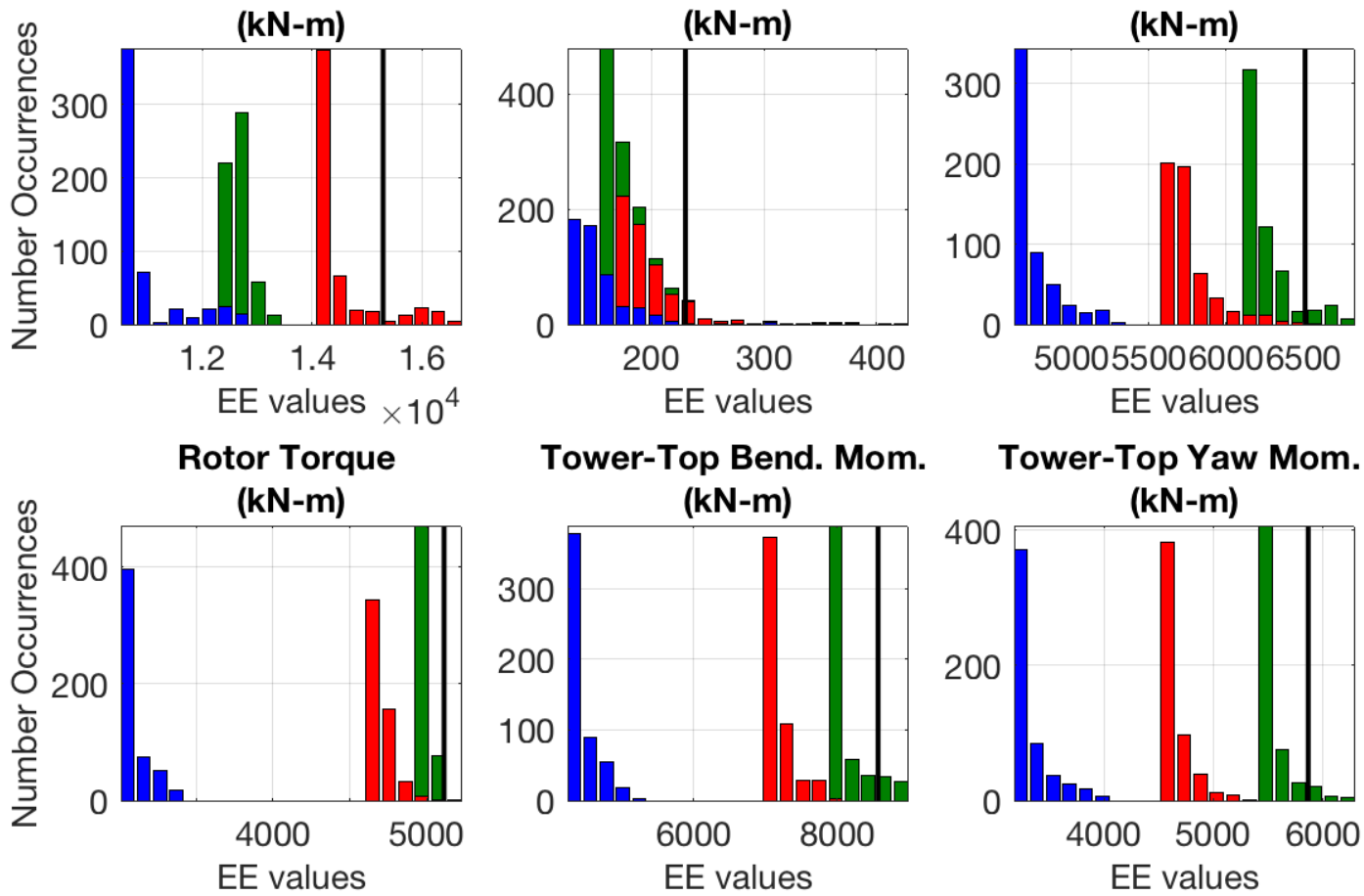

Tower-Top Bend. Mom.

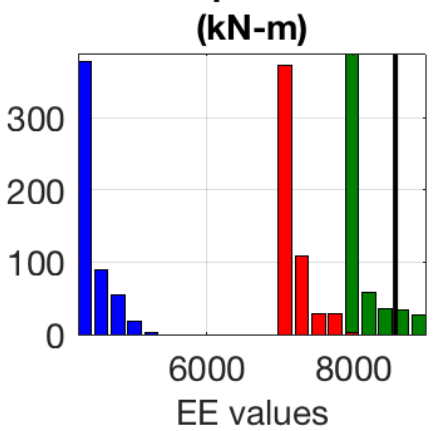

Tower-Top Yaw Mom.

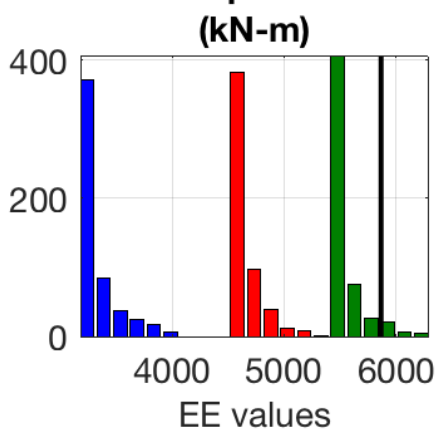

Tower-Base Bend. Mom.

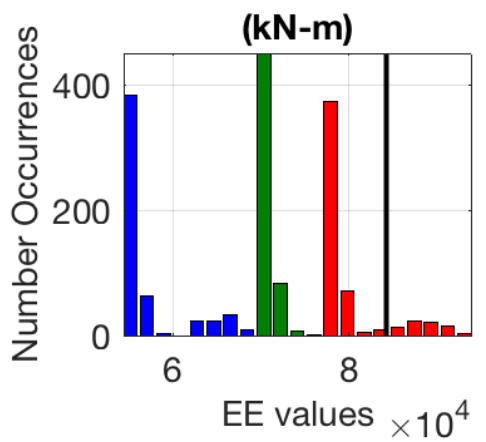

Blade OoP Def.

(m)
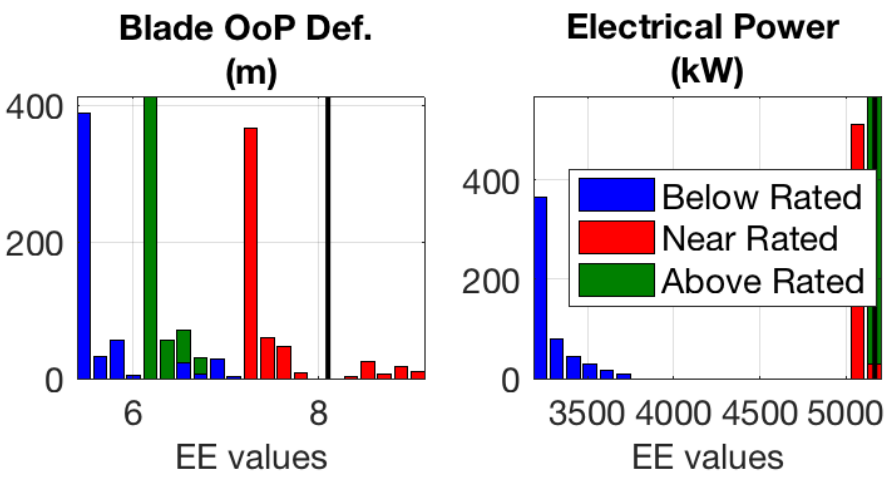

Fig. 7 Stacked histogram of the ultimate load $E E$ values across all wind speed bins, input parameters, and simulation points for all outputs. The black line represents the threshold by which outliers are counted for each output. Color indicates wind speed bin (blue=below rated, red=near rated, green=above rated). 
Blade-Root Bend. Mom.
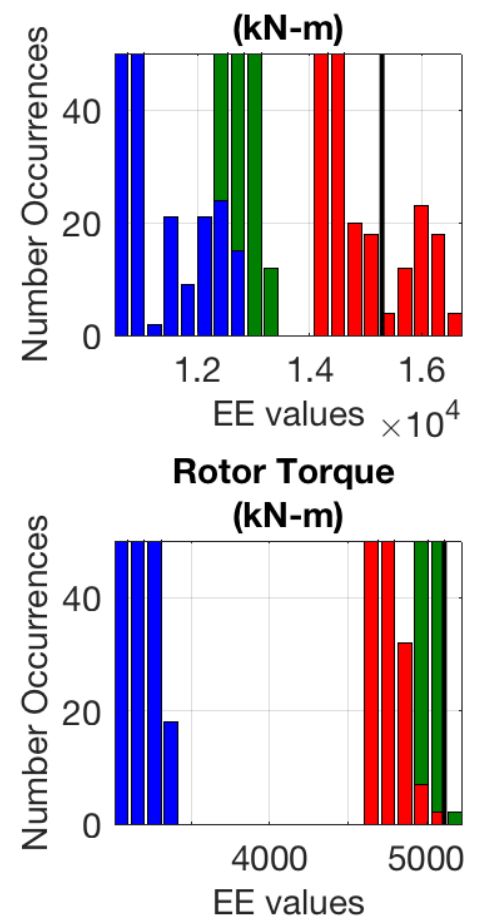

Tower-Base Bend. Mom.

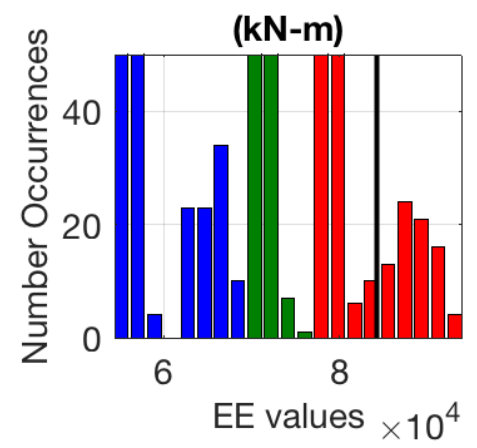

Blade-Root Pitch. Mom.

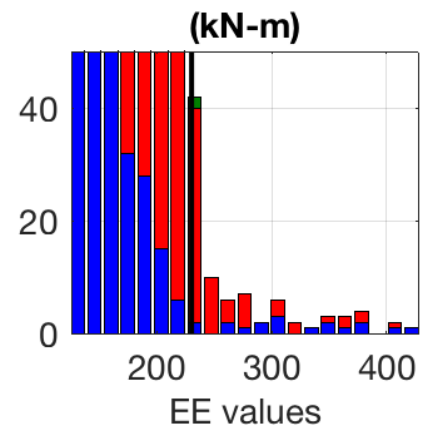

Tower-Top Bend. Mom.

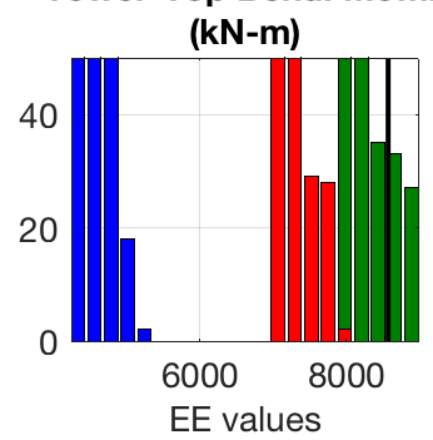

Blade OoP Def. (m)

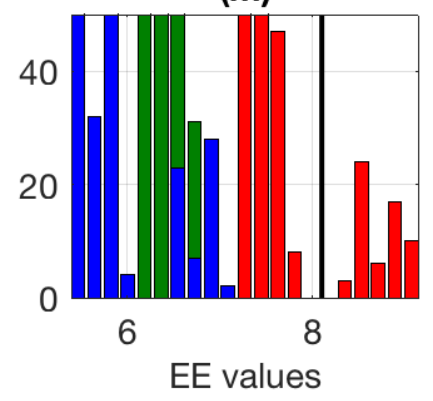

Main Shaft Bend. Mom.

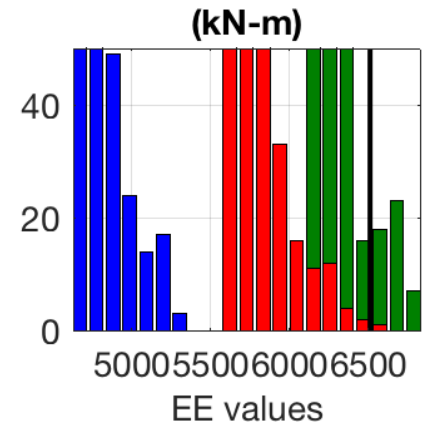

Tower-Top Yaw Mom.

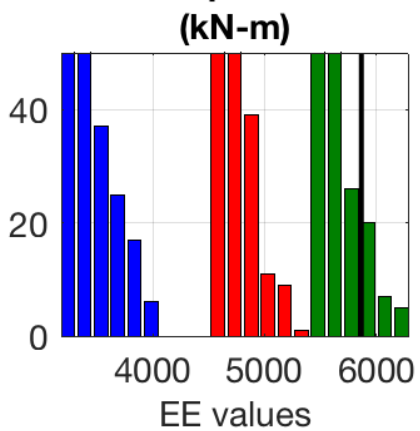

Electrical Power (kW)

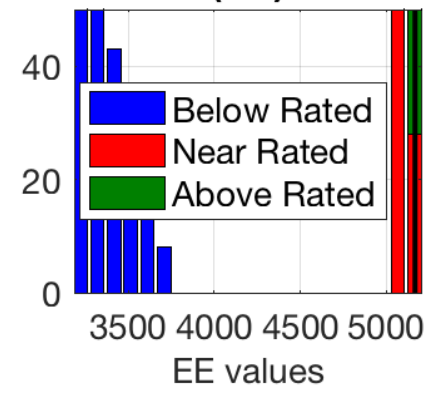

Fig. 8 Zoomed-in stacked histogram of the ultimate load $E E$ values across all wind speed bins, input parameters, and simulation points for all outputs. The black line represents the threshold by which outliers are counted for each output. Color indicates wind speed bin (blue=below rated, red=near rated, green=above rated). 

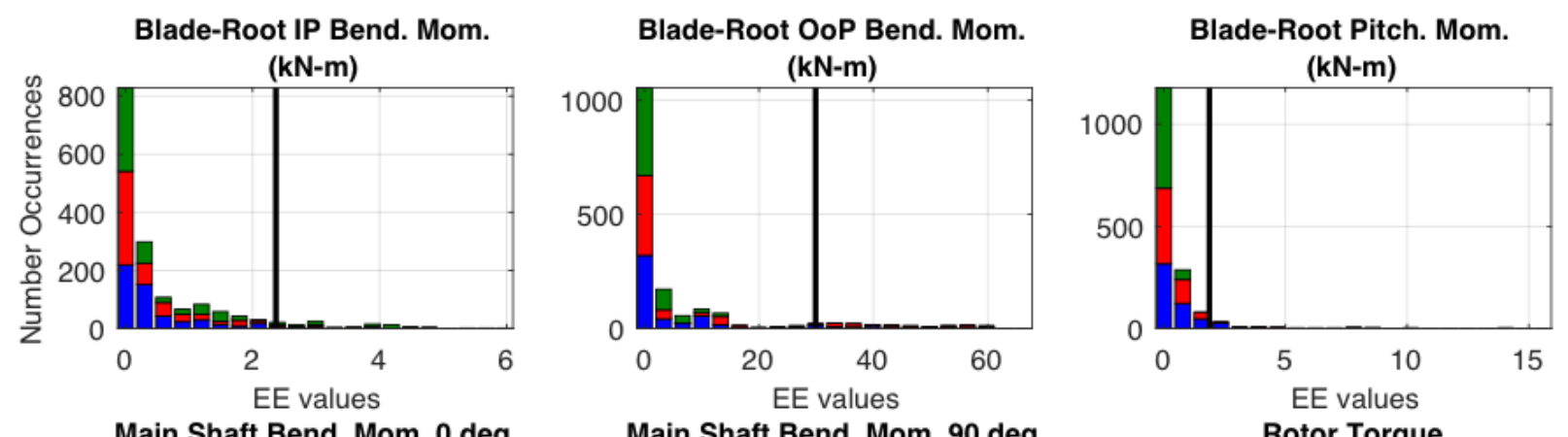

Main Shaft Bend. Mom. 90 deg

Rotor Torque
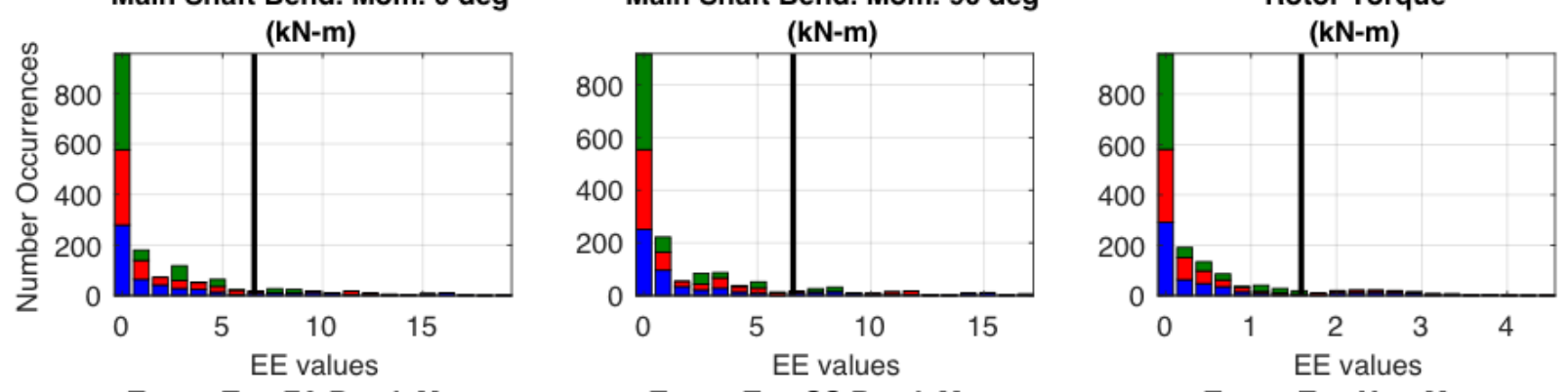

Tower-Top FA Bend. Mom.
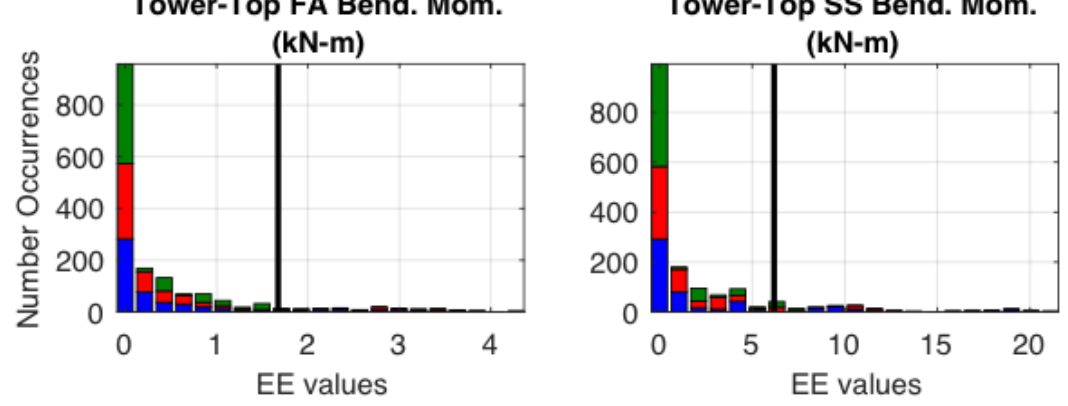

Tower-Top Yaw Mom.

Tower-Base FA Bend. Mom.

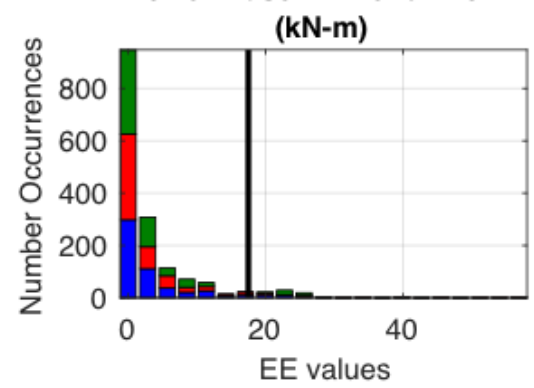

Tower-Base SS Bend. Mom.
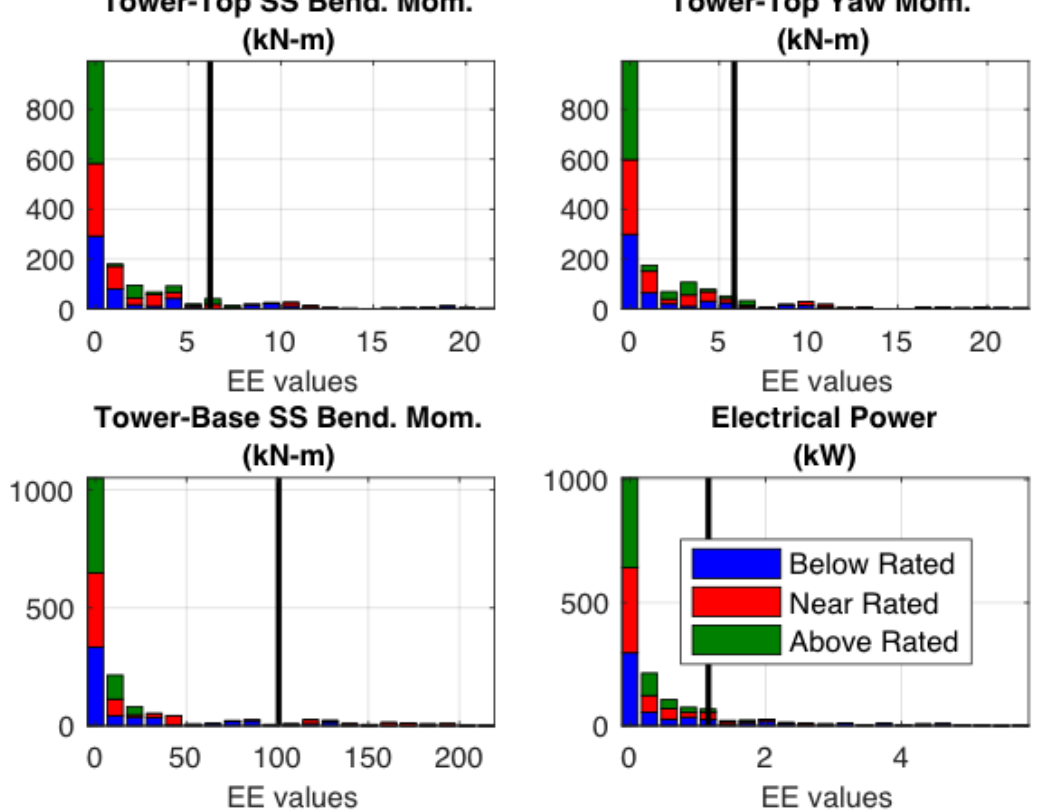

Fig. 9 Stacked histogram of the fatigue load $E E$ values across all wind speed bins, input parameters, and simulation points for all outputs. The black line represents the threshold by which outliers are counted for each output. Color indicates wind speed bin (blue=below rated, red=near rated, green=above rated). 

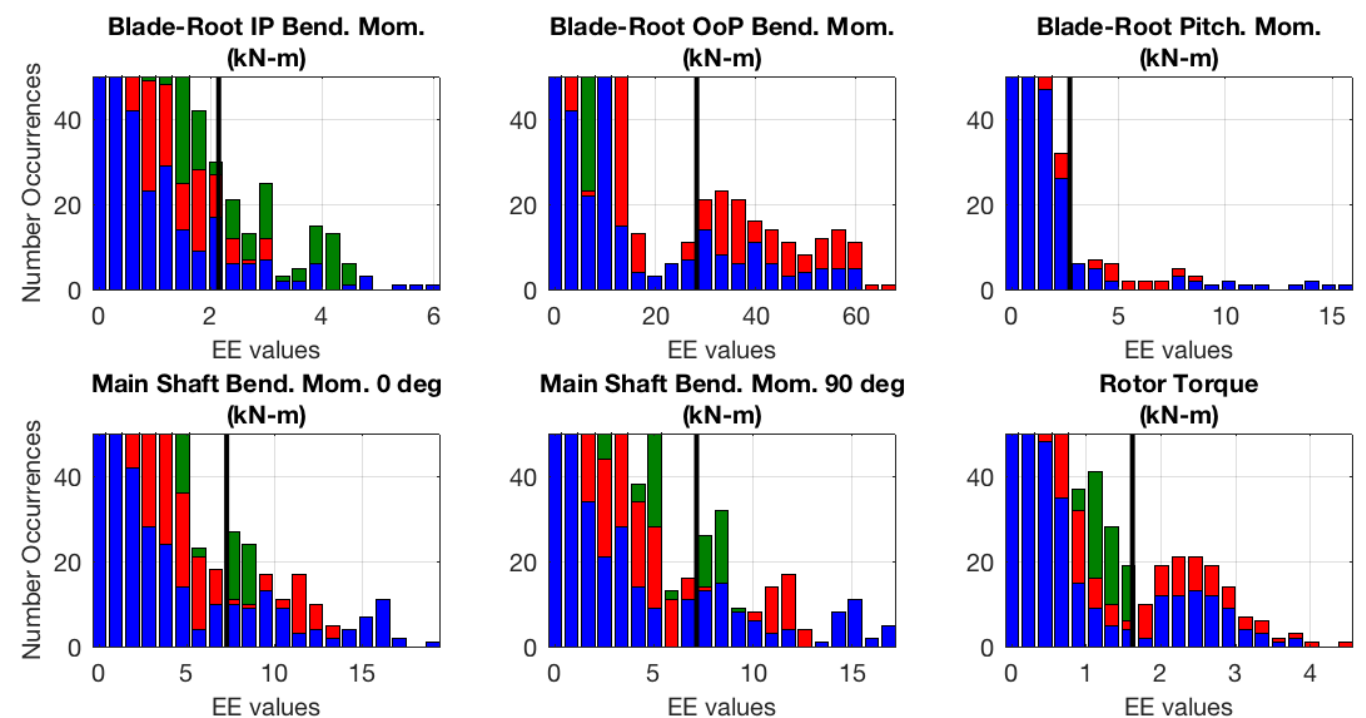

Tower-Top FA Bend. Mom.

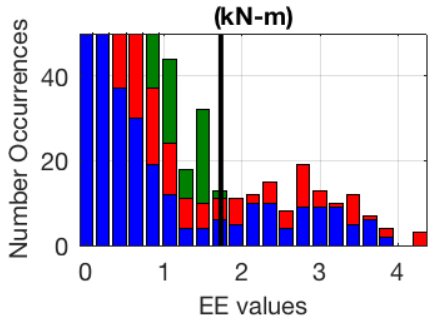

Tower-Top SS Bend. Mom.

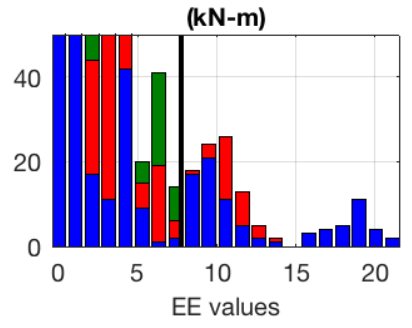

Tower-Top Yaw Mom.

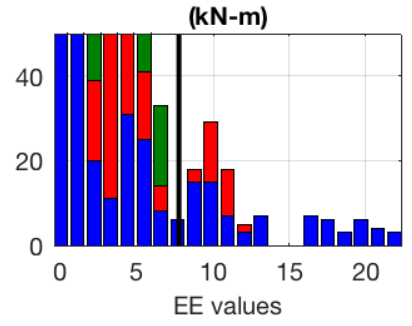

Tower-Base SS Bend. Mom.
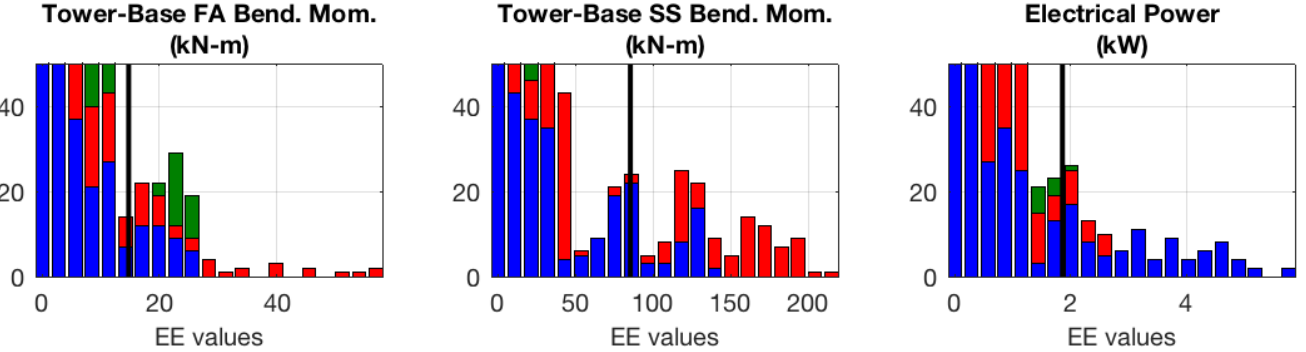

Fig. 10 Zoomed-in stacked histogram of the fatigue load $E E$ values across all wind speed bins, input parameters, and simulation points for all outputs. The black line represents the threshold by which outliers are counted for each output. Color indicates wind speed bin (blue=below rated, red=near rated, green=above rated). 

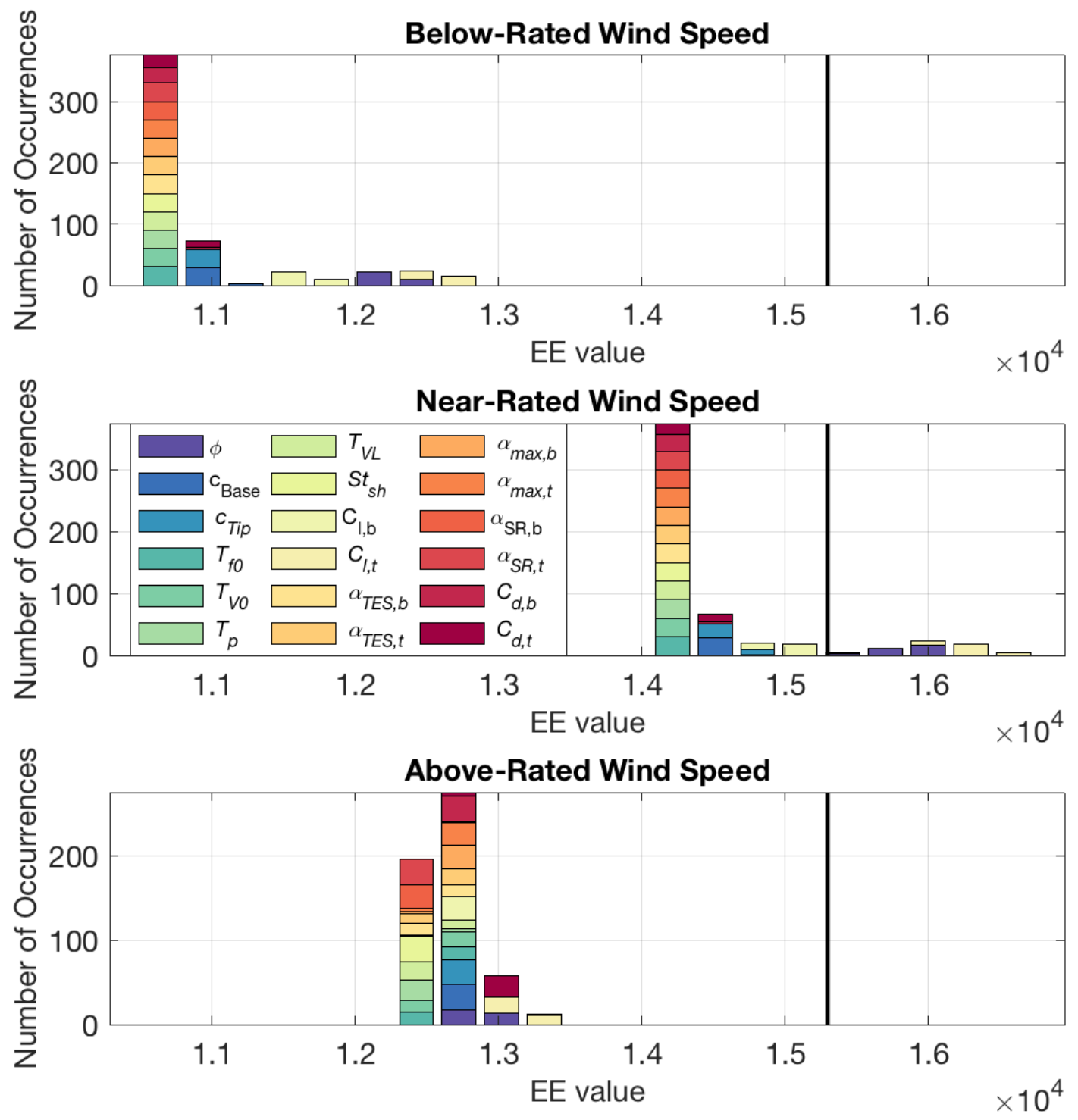

Fig. 11 Histogram of $E E$ values for the blade-root bending ultimate moment. Each graph shows one wind speed bin and includes all input parameters. 

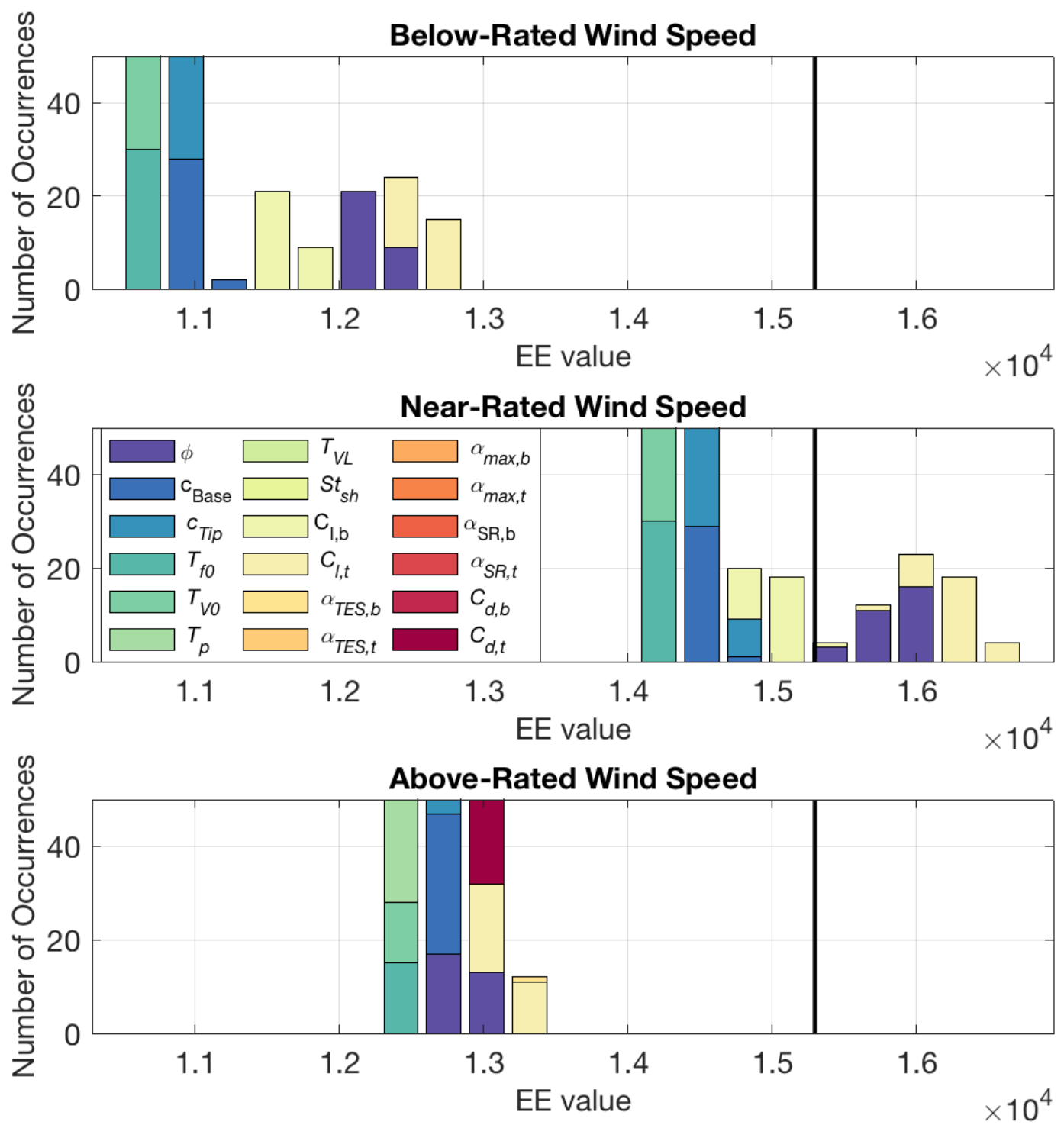

Fig. 12 Zoomed-in histogram of $E E$ values for the blade-root bending ultimate moment. Each graph shows one wind speed bin and includes all input parameters. 

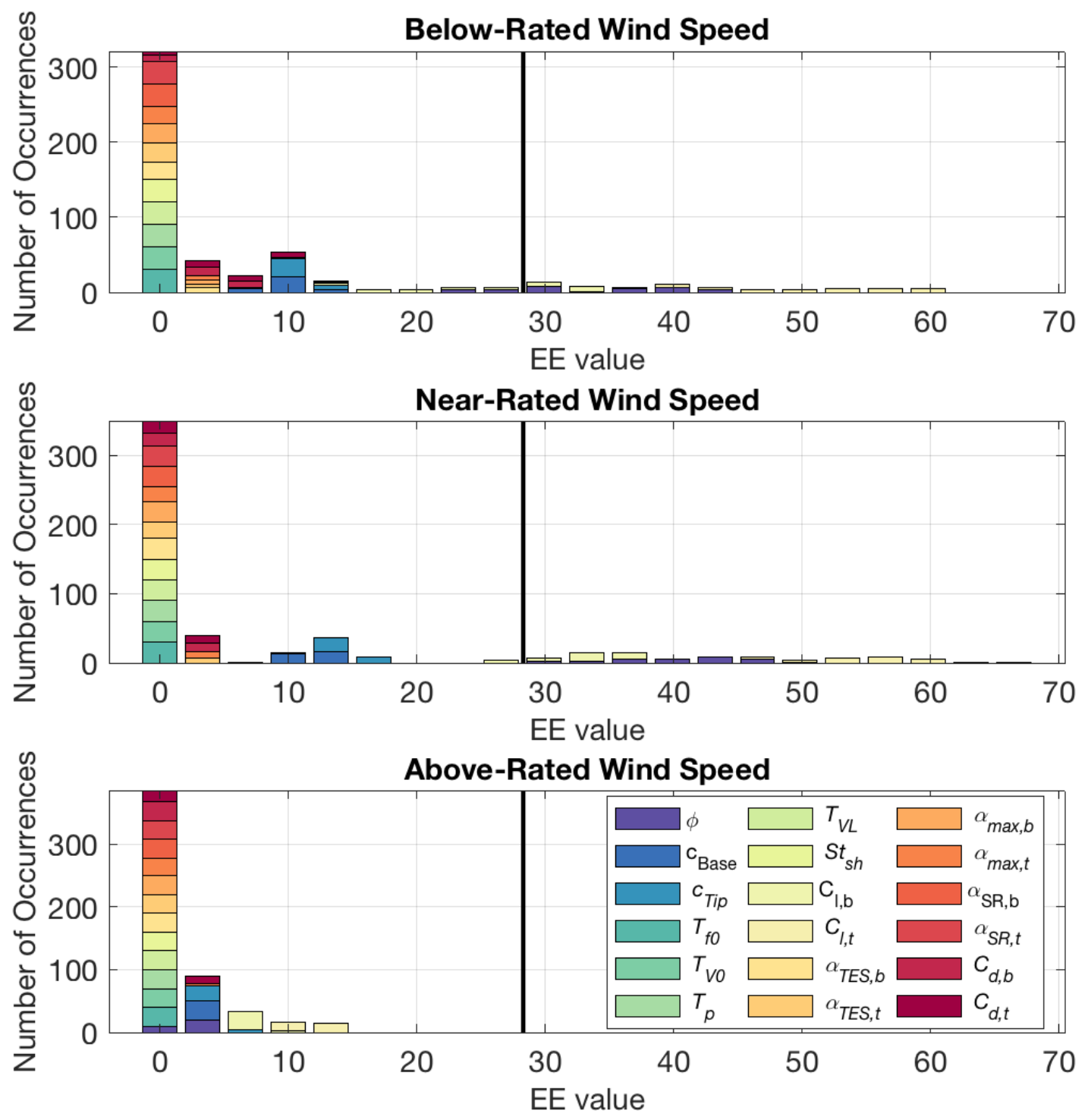

Fig. 13 Histogram of $E E$ values for the blade-root OoP bending fatigue moment. Each graph shows one wind speed bin and includes all input parameters. 

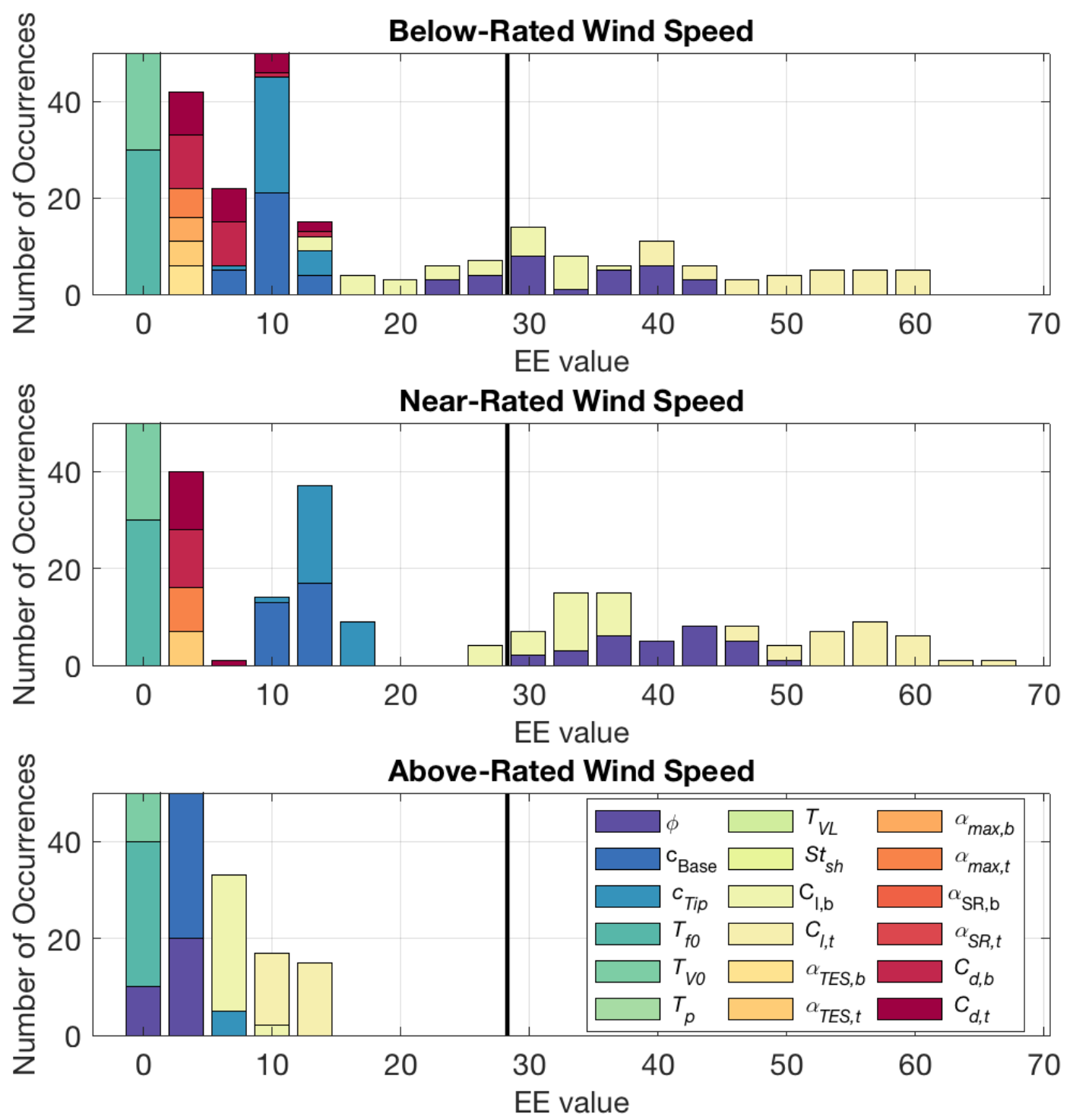

Fig. 14 Zoomed-in histogram of $E E$ values for the blade-root out-of-plane bending fatigue moment. Each graph shows one wind speed bin and includes all input parameters. 

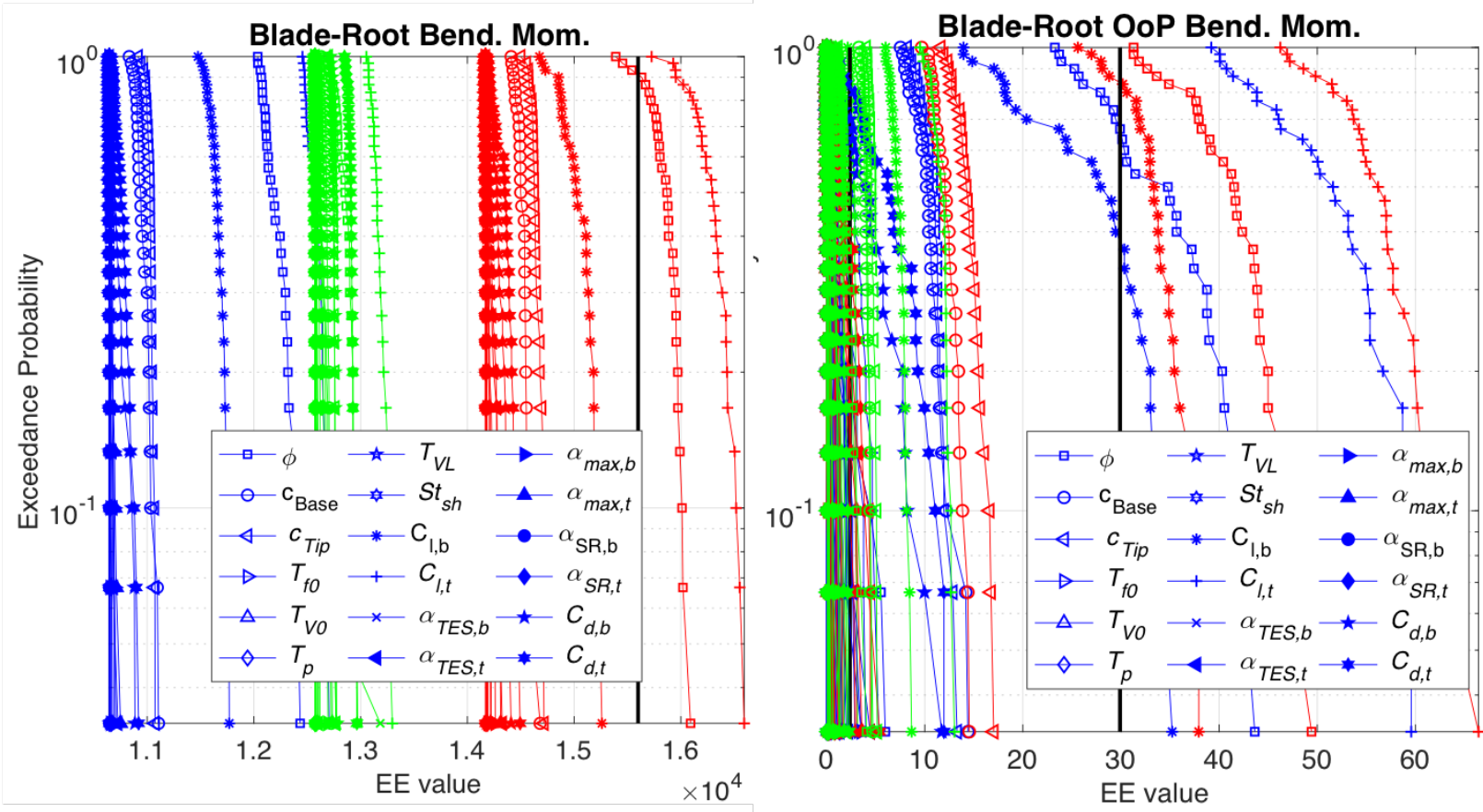

Fig. 15 Exceedance probability plot of $E E$ values for the blade-root bending ultimate moment (left) and bladeroot out-of-plane bending fatigue moment. Each line represents a different input parameter and wind speed bin (blue=below rated, red=near rated, green=above rated).

\section{Conclusion}

The purpose of the study was to assess the sensitivity of different turbine parameters on the resulting loads of the wind turbine. The sensitivities of the different parameters were ranked; in future work, this information can be used to help establish error bars around predictions of engineering models during validation efforts and provide insight to probabilistic design methods and site-suitability analyses. This study shows that the loads and power are highly sensitive to the $C_{l}$ and blade twist distribution. To a lesser extent, turbine loads are sensitive to turbine blade chord distribution, $\alpha_{\max , b}$, and $C_{d}$ distributions. Additionally, ultimate load $E E$ values are typically separated by wind speed bin, whereas fatigue load $E E$ values are more evenly distributed across wind speed bins. It was also identified that, when an input parameter contributes to the outlier count for a given output parameter, it is possible that only certain hyperspace locations lead to the threshold exceedence. This leads to opportunities for future work to further investigate which hyperspace locations lead to higher turbine sensitivity.

\section{Acknowledgments}

This work was authored by the National Renewable Energy Laboratory, operated by Alliance for Sustainable Energy, LLC, for the U.S. Department of Energy (DOE) under Contract No. DE-AC36-08GO28308. Funding provided by the U.S. Department of Energy Office of Energy Efficiency and Renewable Energy Wind Energy Technologies Office. The views expressed in the article do not necessarily represent the views of the DOE or the U.S. Government. The U.S. Government retains and the publisher, by accepting the article for publication, acknowledges that the U.S. Government retains a nonexclusive, paid-up, irrevocable, worldwide license to publish or reproduce the published form of this work, or allow others to do so, for U.S. Government purposes.

\section{References}

[1] Robertson, A., Sethuraman, L., Jonkman, J., and Quick, J., "Assessment of Wind Parameter Sensitivity on Ultimate and Fatigue Wind Turbine Loads,” AIAA SciTech Forum, AIAA, Grapevine, TX, 2018, pp. 1-23. doi:10.2514/6.2016-1007.

[2] Abdallah, G., Natarajan, A., and Sorensen, J., "Impact of Uncertainty in Airfoil Characteristics on Wind Turbine Extreme 
Loads,” Renewable Energy, Vol. 75, 2015, pp. 283-300. doi:10.1016/j.renene.2014.10.009.

[3] Simms, D., Schreck, S., Hand, M., and Fingersh, L., "NREL Unsteady Aerodynamics Experiment in the NASA-Ames Wind Tunnel: A Comparison of Predictions to Measurements," Tech. Rep. NREL/TP-500-29494, National Renewable Energy Laboratory, Golden, CO, June 2001.

[4] Madsen, H. A., Bak, C., Paulsen, U. S., Gaunaa, M., Fuglsang, P., and Romblad, J., "The DAN-AERO MW Experiments Final Report,” Tech. Rep. Riso-R-1726(EN), Riso DTU, Roskilde, Denmarkß, September 2010.

[5] Damiani, R. R., Hayman, G. J., and Jonkman, J., "Development and Validation of a New Unsteady Airfoil Aerodynamics Model Within AeroDyn,” AIAA SciTech Forum, AIAA, San Diego, CA, 2016, pp. 1-21. doi:10.2514/6.2016-1007.

[6] Gan, Y., Duan, Q., Gong, W., Tong, C., Sun, Y., and Chu, W., “A Comprehensive Evaluation of Various Sensitivity Analysis Methods: A Case Study with a Hydrological Model,” Environmental Modelling and Software, Vol. 51, 2014, pp. $269-285$.

[7] Martin, R., Lazakis, I., Barbouci, S., and Johanning, L., "Sensitivity Analysis of Offshore Wind Farm Operation and Maintenance Cost and Availability," Renewable Energy, Vol. 85, 2016, pp. 1226-1236.

[8] Saranyasoontorn, K., and Manuel, L., "On the Study of Uncertainty in Inflow Turbulence Model Parameters in Wind Turbine Applications," 44th AIAA Aerospace Sciences Meeting and Exhibit, AIAA, Reno, NV, 2006. doi:10.2514/6.2016-1007.

[9] Jonkman, J., Butterfield, S., Musial, W., and Scott, G., "Definition of a 5-MW Reference Wind Turbine for Offshore System Development,” Tech. Rep. NREL/TP-500-38060, National Renewable Energy Laboratory, Golden, CO, February 2009.

[10] “OpenFAST Documentation,”, November 2017. URL http://openfast.readthedocs.io/en/master/

[11] Hayman, G. J., "MLife Theory Manual for Version 1.00," Tech. Rep. NREL/TP-XXXXX, National Renewable Energy Laboratory, Golden, CO, October 2012.

[12] Jonkman, B., "TurbSim User's Guide v2.00.00,” Tech. Rep. NREL/TP-xxxx-xxxxx, National Renewable Energy Laboratory, Golden, CO, October 2014.

[13] Damiani, R., personal communication, May 2018.

[14] Petrone, G., de Nicola, C., Ouagliarella, D., and Witteveen, J. A. S., "Wind Turbine Performance Analysis Under Uncertainty," AIAA SciTech Forum, AIAA, 2011.

[15] Loeven, G. J. A., and Bihl, H., "Airfoil Analysis with Uncertain Geometry Using a Probabilistic Collection Method," AIAA Structures, Structural Dynamics, and Materials Conference, 2008.

[16] Ehrmann, R. S., Wilcox, B., White, E. B., and et al., "Effect of Surface Roughness on Wind Turbine Performance," Tech. Rep. SAND2017-10669, Sandia National Laboratories, Albuquerque, NM, October 2017.

\section{A. Mean and Standard Deviation of Elementary Effects}

As commonly found in $E E$-related literature, $E E$ analysis typically identifies the most sensitive parameters using a plot to pictorially show the standard deviation versus mean values of the $E E$ values. The mean of the absolute Elementary Effects value for the ultimate loads for output $o$, input parameter $i$, and bin $b$ is calculated as:

$$
\mu_{\text {oib }}^{*}=\frac{1}{R} \sum_{r=1}^{R}\left|E E_{\text {oib }}^{r}\right|
$$

where $R$ is the number of points at which the $E E$ value is calculated. The standard deviation of the elementary effect is then calculated as:

$$
\sigma_{o i b}=\sqrt{\frac{1}{R-1} \sum_{r=1}^{R}\left(E E_{o i b}^{r}-\mu_{o i b}\right)^{2}}
$$

and $\mu_{o i b}$ is defined as:

$$
\mu_{o i b}=\frac{1}{R} \sum_{r=1}^{R} E E_{o i b}^{r}
$$

This is shown in Figure 16 for the blade-root bending ultimate moment and for the blade-root bending OoP fatigue moment metrics. These plots show the large sensitivity of $C_{l, t}, \phi$, and $C_{l, b}$ in the near-rated wind speed bin, as well as the separation of wind speed bin results for ultimate load $E E$ values. 

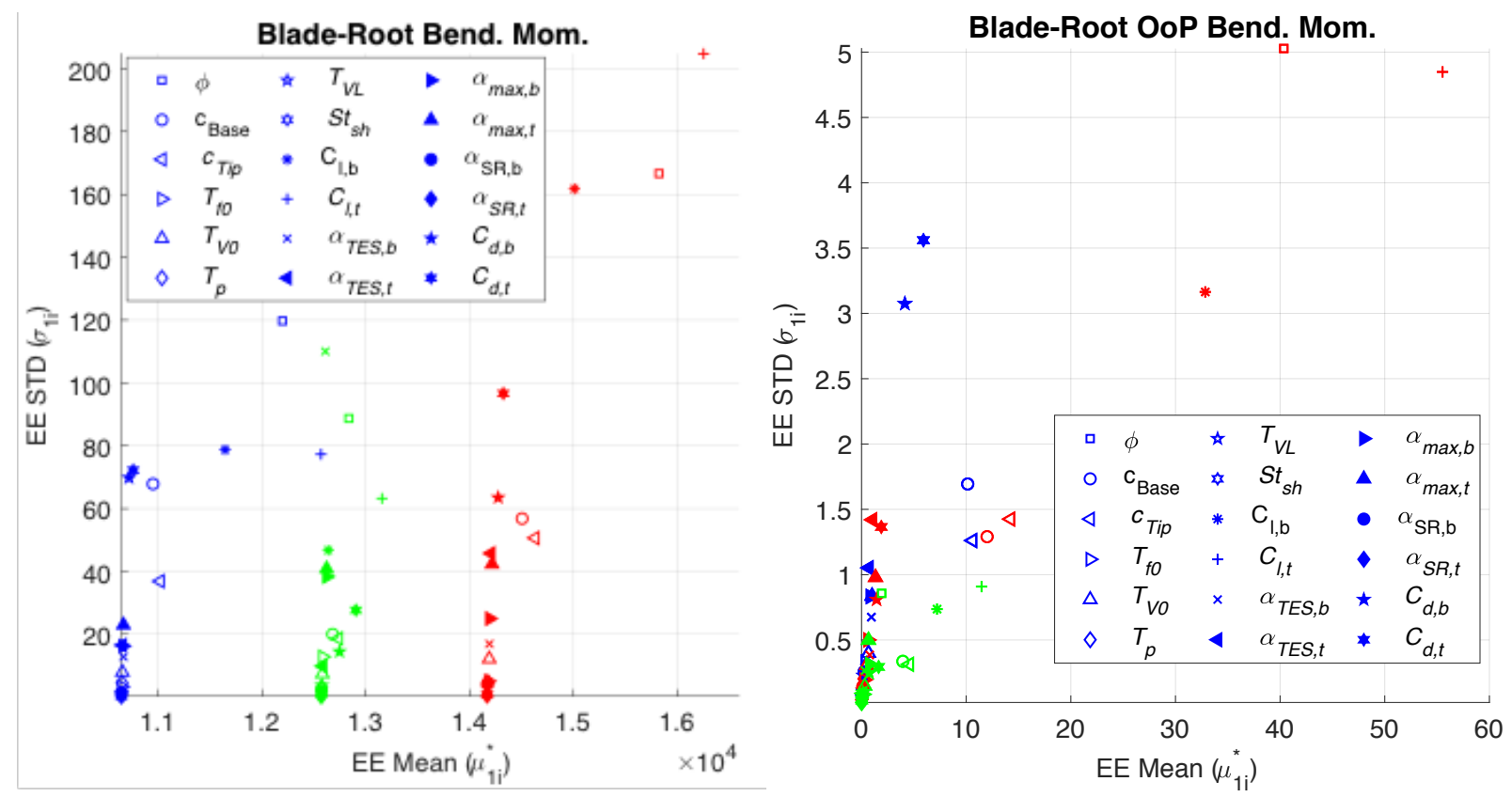

Fig. $16 E E$ standard deviation vs $E E$ mean value for blade-root bending ultimate moment (left) and bladeroot out-of-plane bending fatigue moment (right) at all wind speed bins (blue=below rated, red=near rated, green=above rated). 